\title{
Notizbuch eines Beamten der Metropolis in Thessalonike aus dem Anfang des $\mathbf{X W}$. Jahrhunderts.
}

\section{$\S 1$. Überlieferung.}

Die unten veröffentlichten Notizen habe ich vor vier Jahren in Paris aus cod. Graecus 2953 der Bibliothèque Nationale abgeschrieben. Dieser Kodex, eine Bombycinhs des XIIII. Jahrh:1), enthält zum gröBten Teil Reden. des Rhetors Aristides mit Scholien. Anfang ( $\Pi \alpha \nu \alpha \vartheta \eta v \alpha i x o ́ s)$

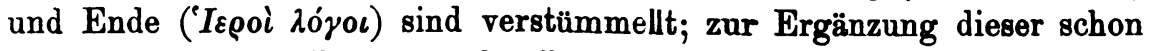
früh entstandenen Lücke sind offenbar bei der ziemlich alten Buchbindung im Anfang und am Ende des Bamdes 16 unbeschriebene Papierblätter zugefügt worden. Diese Blätter hat jedoch später ein Besitzer des Kodex zur Eintragung seiner persøinlichen Notizen benutzt; der gröBte Teil von ihnen wird hier unten mitgeteilt. Ich gebe den Text dieses Notizbuches genau wieder und in derselben Reihe, in der die Notizen im Kodex sich folgen; zur Erleichterung der Hinweise habe ich die Notizen numeriert. Einige weynige und ganz unbedeutende Schreibfehler habe ich stillschweigend korrigiert. Fast alle diese Notizen sind, zu verschiedenen Zeiten und mit Benutzung verschiedener Tinten, von ein und derselben Hand geschrieben worden, von der auch viele Scholien und Randnotizen in Aristidestext herrühren. ${ }^{2}$ ) Die flüchtige, durchaus nicht sorgsame Schrift verrät einen gelehrten Schreiber; auBer den gewöhnlichen Abkürzungen sind tachygraphische Zeichen fur

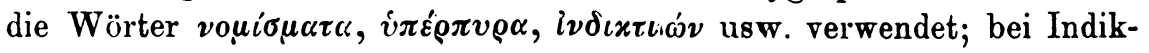
tions- oder Jahreszahlen ist auf jeden Zaublbuchstaben die Endung der Ordnungszahl (-ov, - $\eta$ s usw.) gesetzt. Jede Notiz beginnt und schlieBt mit einem Kreuzzeichen; auch die an der Spitze stehende Anrufung Christi (Nr. 1) zeigt fromme Tradition. Dras häufige Durchstreichen und Korrigieren bei einzeluen Wörtern verräit den flüchtigen und streng persönlichen Charakter der Notizen.

1) H. Omont, Inventaire sommaire des mamuscr. Grecs de la Bibl. Nationale Bd. III S. $69 \mathrm{ff}$.

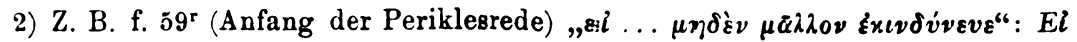

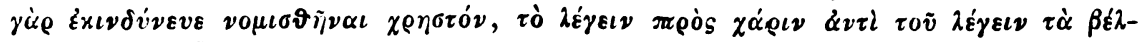

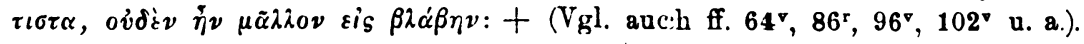


§ 2. Text.

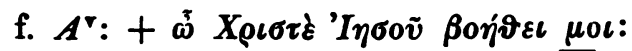

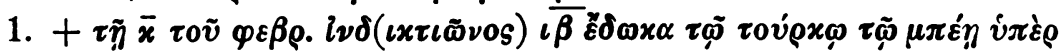

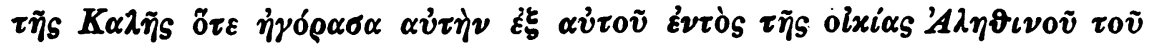

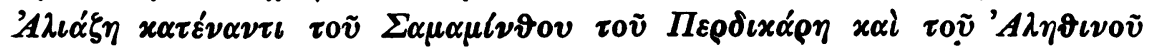

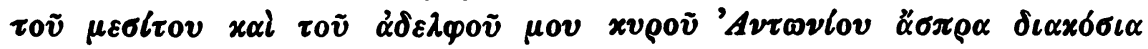

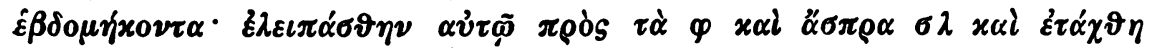

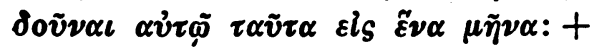

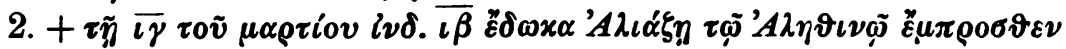

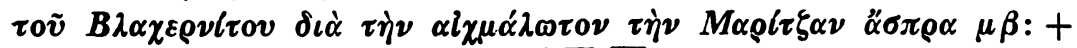

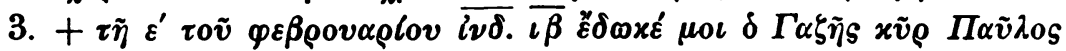

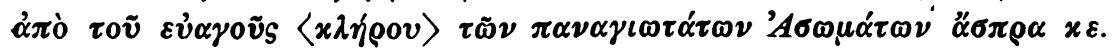

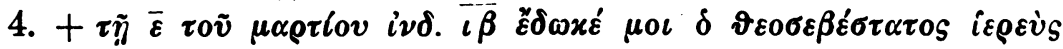

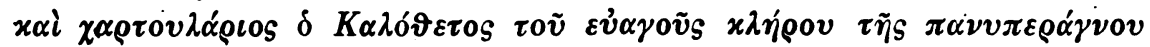

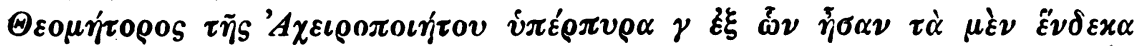

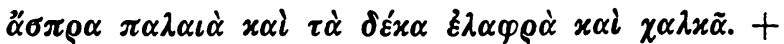

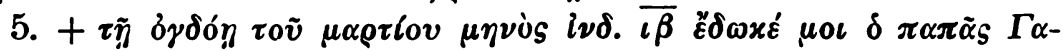

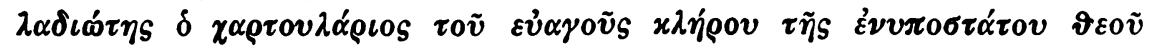

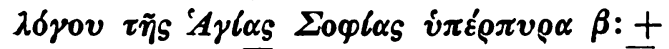

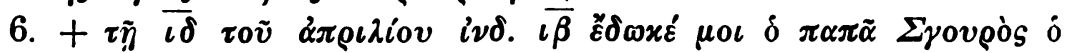

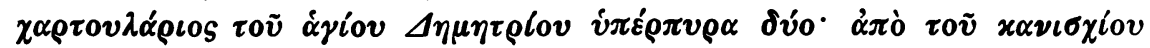

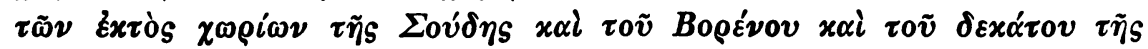

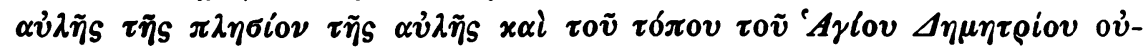

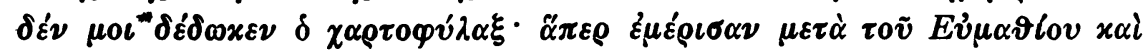

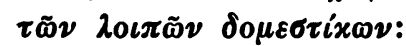

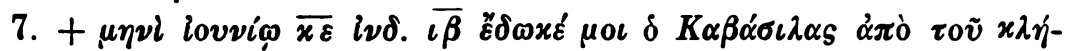

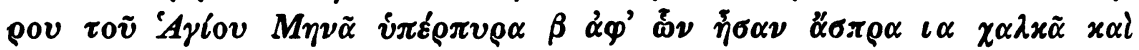

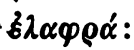

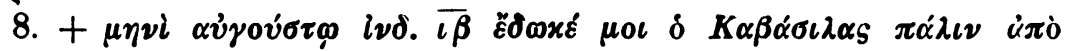

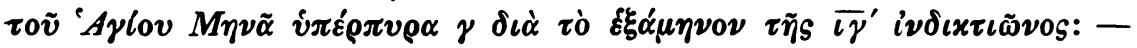

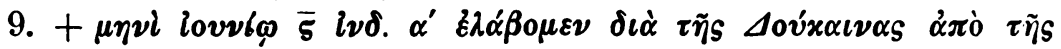

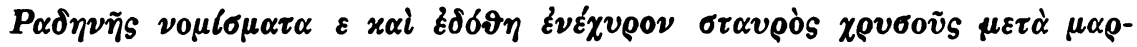

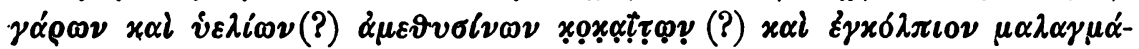

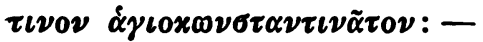

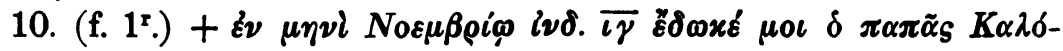

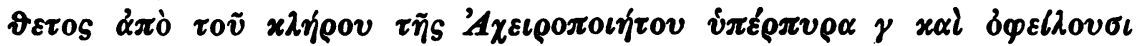

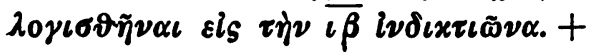

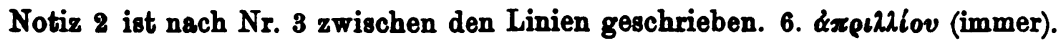

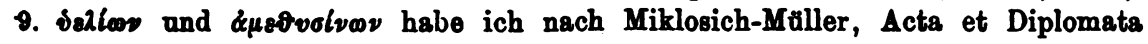
Bd. I S. 686; II S. 666-567 gelesen. 


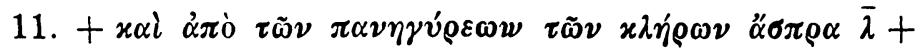

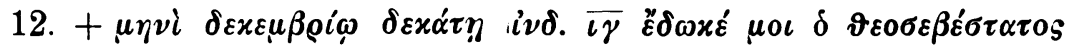

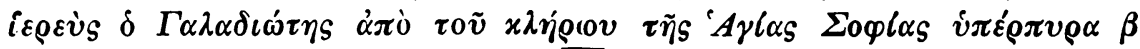

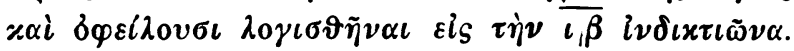

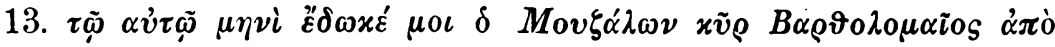

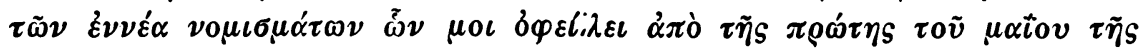

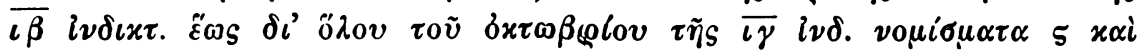

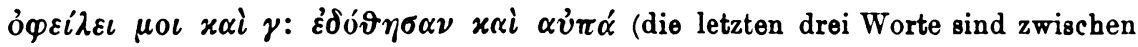
den Linien geschrieben).

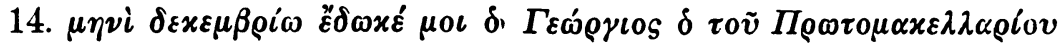

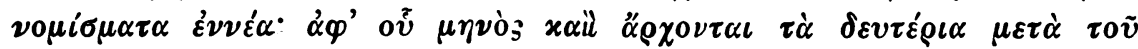

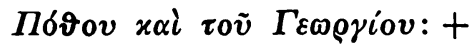

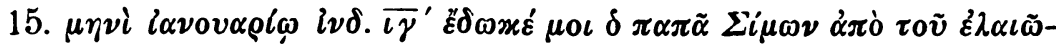

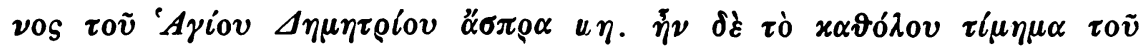
$\dot{\varepsilon} \lambda \alpha \iota \tilde{\omega} \nu 0 \mathrm{~S} \nu 0 \mu l \sigma \mu \alpha \tau \alpha \xi$.

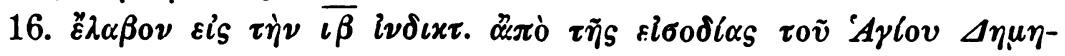

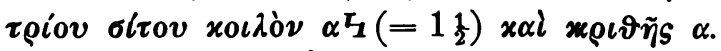

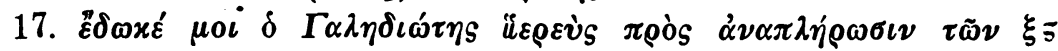

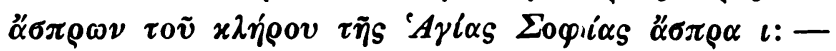

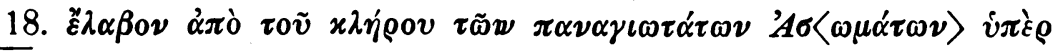

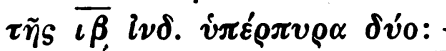

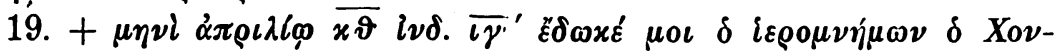

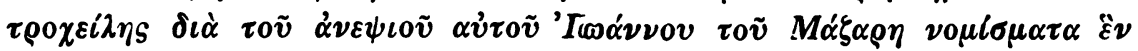

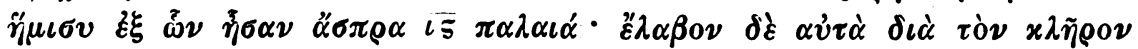
$\tau o \tilde{v}{ }^{`} A \gamma i ́ o v M r v \tilde{\alpha}+x \alpha i \alpha \tilde{v} \vartheta \iota \varsigma \sigma \tau \alpha w \rho \tilde{\alpha} \tau \alpha \beta$ (die letzten drei Worte durchgestrichen).

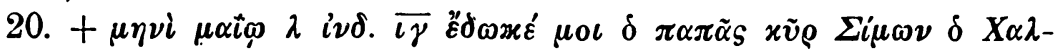

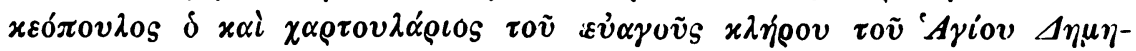

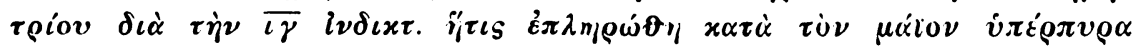
$\mu o ́ v \alpha \beta$.

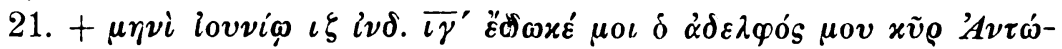

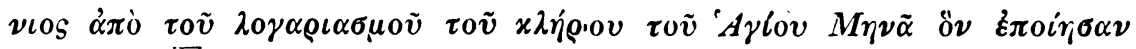

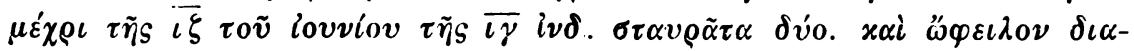

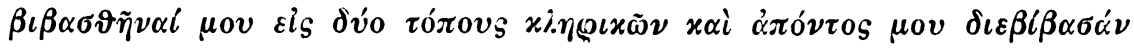

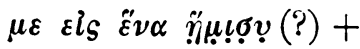

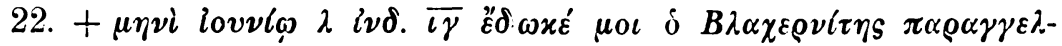

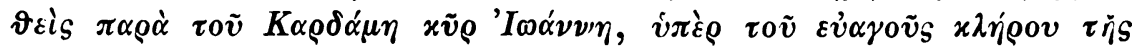

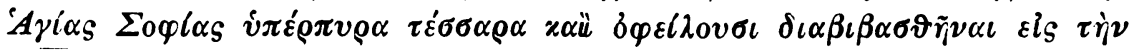

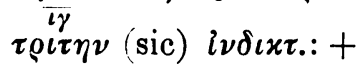

12. $\delta \varepsilon x \varepsilon \beta \rho i \omega$ (immer).

Byzant. Zeitschrift XXIII 1 u. 2 . 


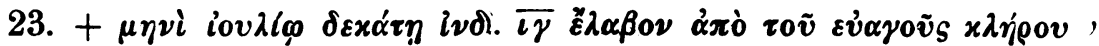

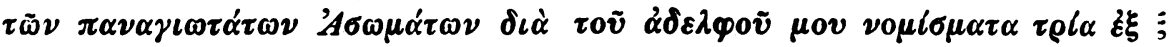

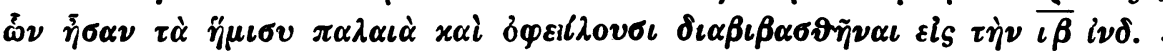

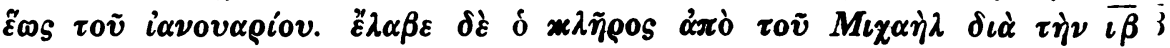

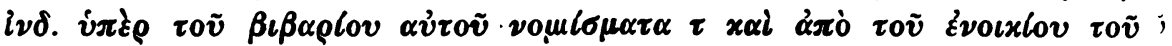

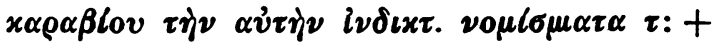

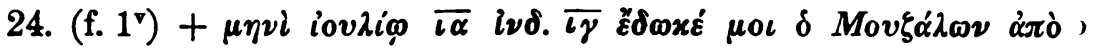

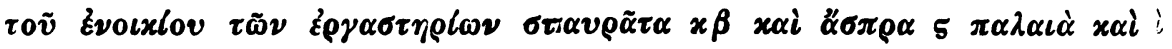

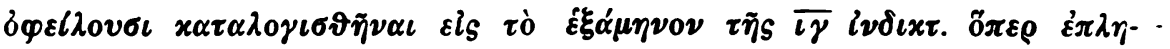

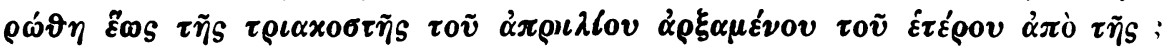
$\alpha^{\prime} \tau 0 \tilde{v} \mu \alpha \hat{i} 0 v$.

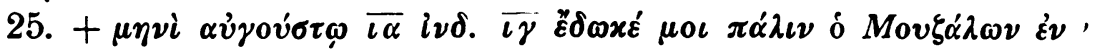

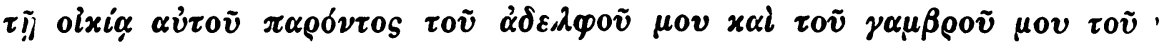

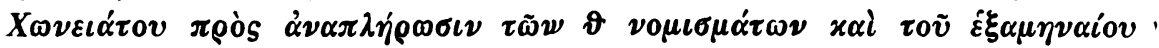

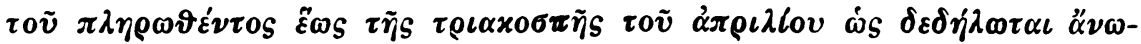

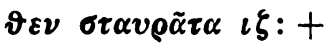

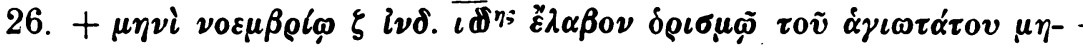

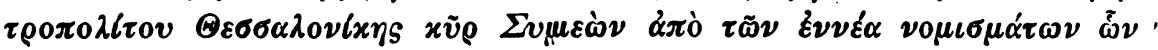

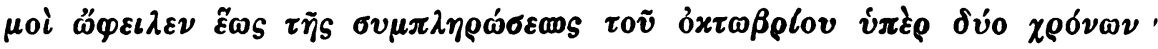

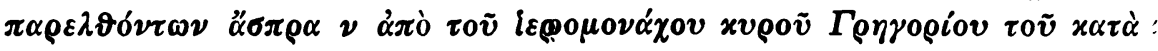

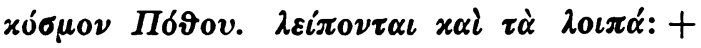

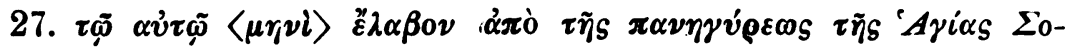

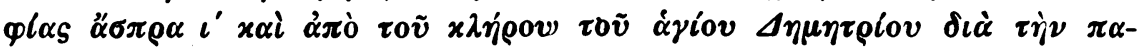
$\nu \eta^{\prime} \gamma v \rho \iota \nu \ddot{\alpha} \sigma \pi \rho \alpha \iota \beta$.

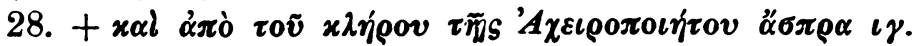

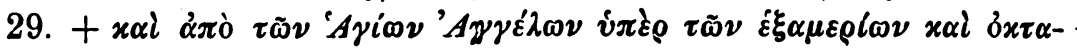

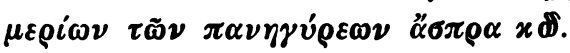

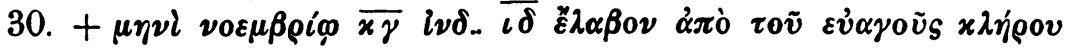

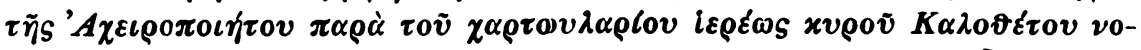

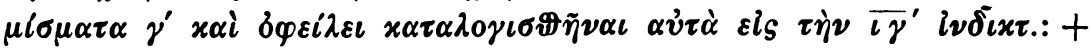

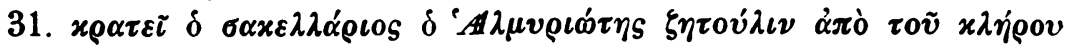

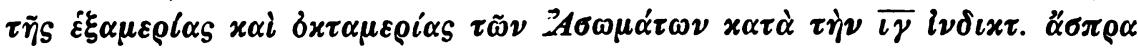

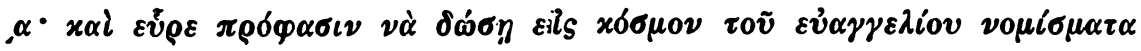

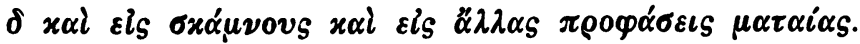

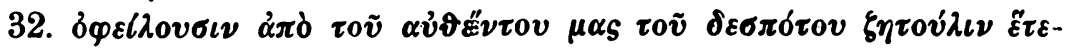

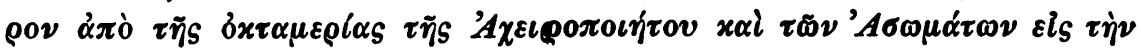

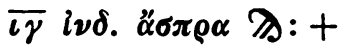

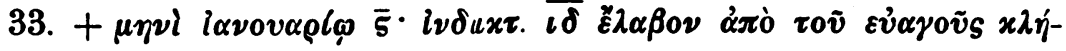

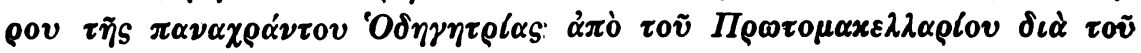

23. lovגaí (immer). 


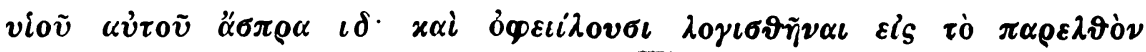

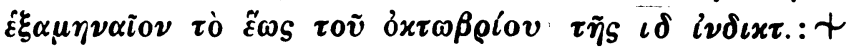

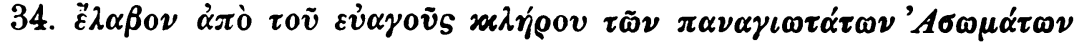

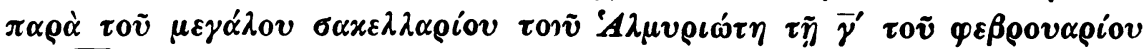

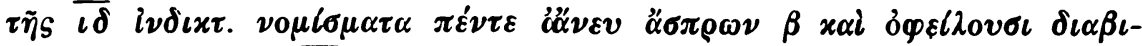

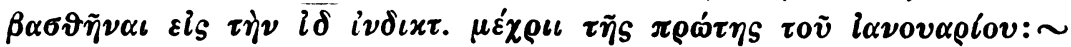

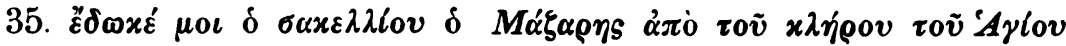

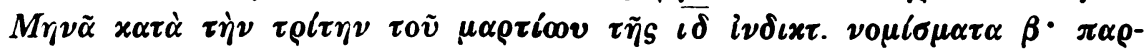

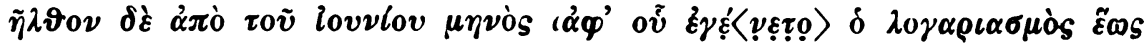

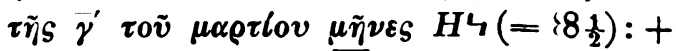

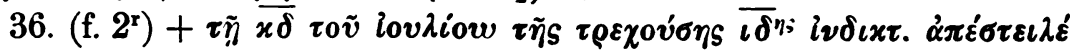

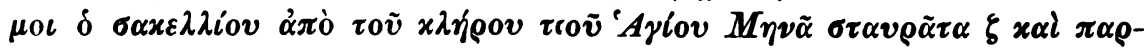

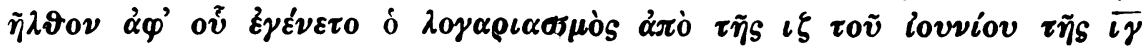

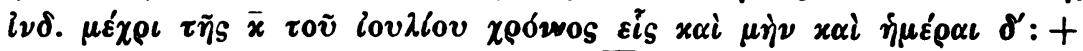

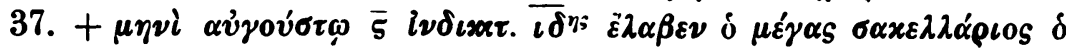

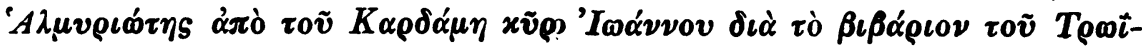

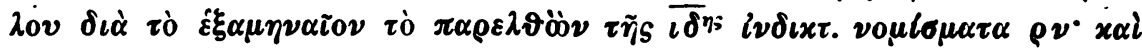

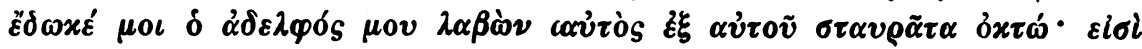
$\delta \dot{\varepsilon}$ oi $x \lambda \eta \varrho \iota x o i ~ \delta \iota \dot{\alpha} \pi \alpha \dot{\alpha} \nu \tau \omega \nu \mu:+$

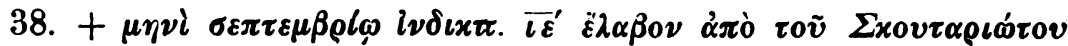

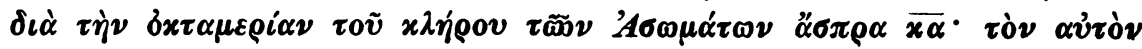

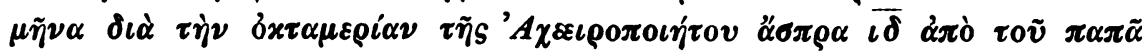

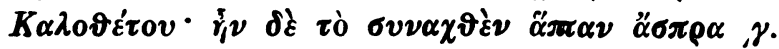

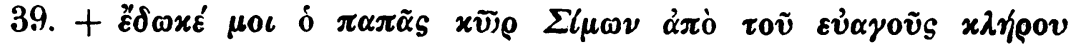

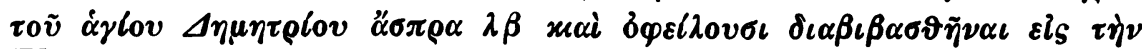

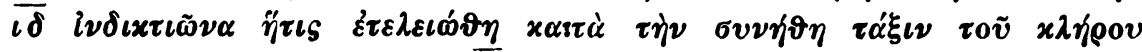

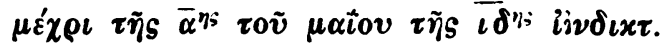

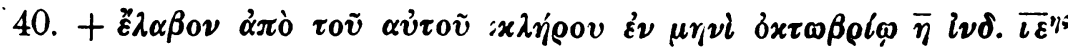

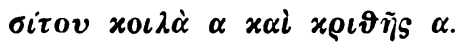

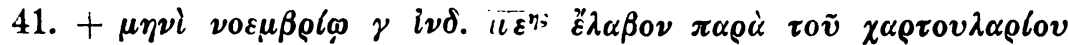

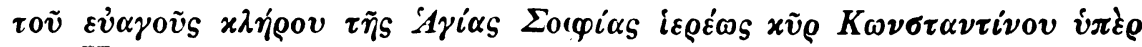

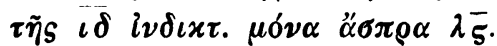

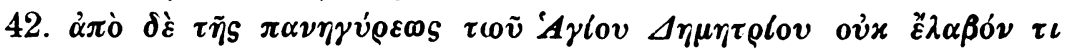

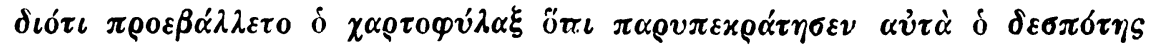

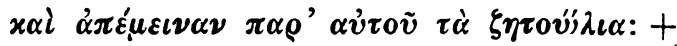

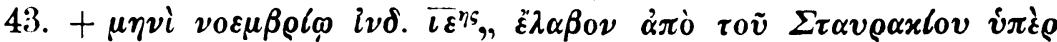

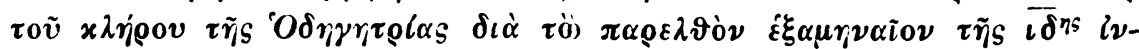
$\delta \iota x \tau$. $\ddot{x} \sigma \pi \rho \alpha \overline{\iota \delta}$.

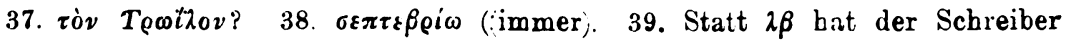
früher $x \alpha$ geschrieben. 


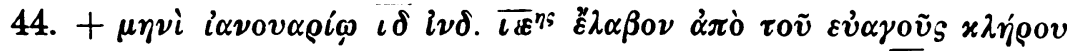

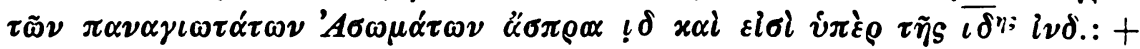

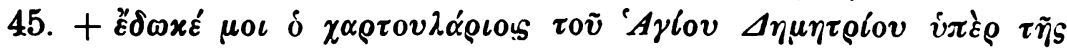
¿ठ"

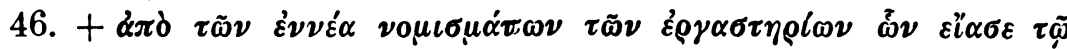

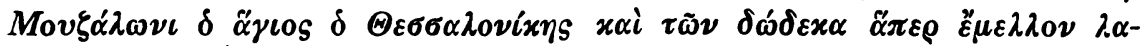

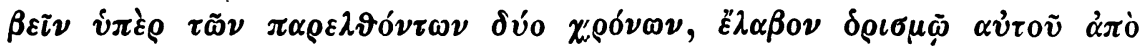

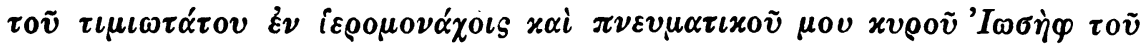
$K v \beta$ ov

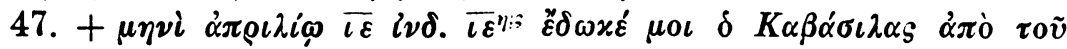

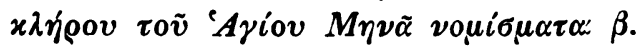

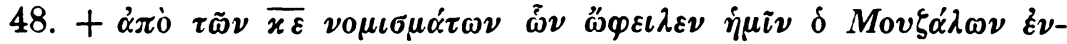

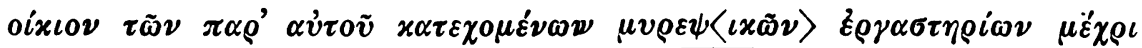

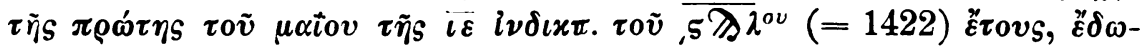

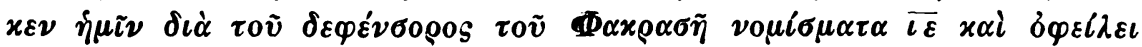

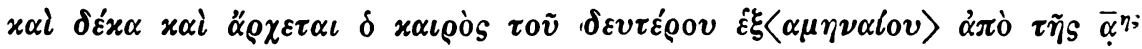

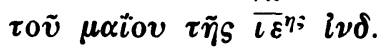

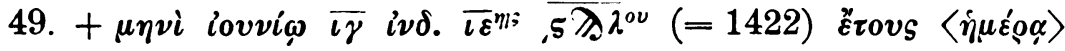

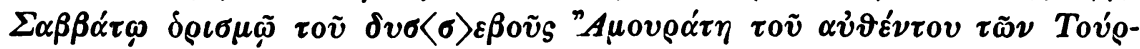

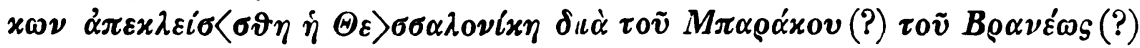

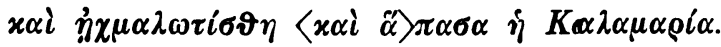

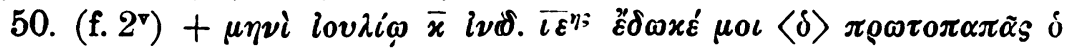

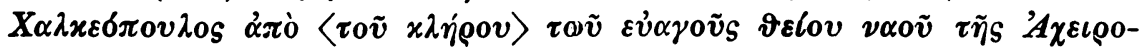

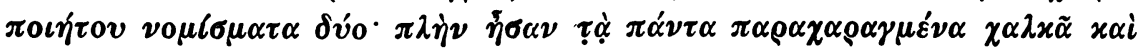

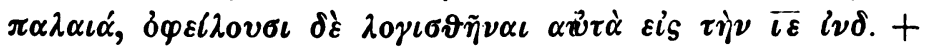

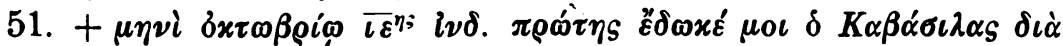

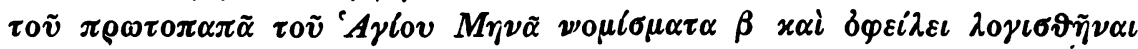

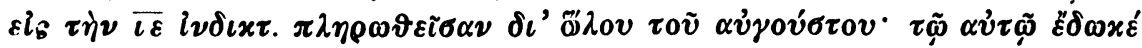

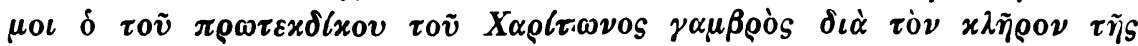

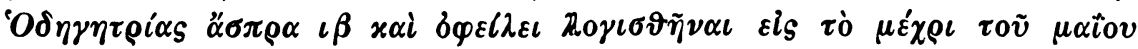
$\varepsilon \xi \alpha \mu \eta \nu \alpha i o \nu \tau \tilde{\eta} s \bar{\imath}^{\eta ;} i \nu \delta \iota x \tau .:+$

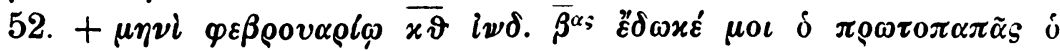

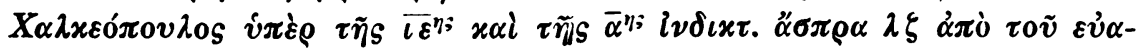

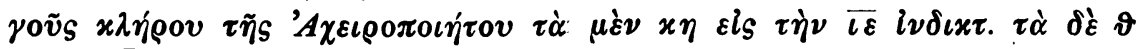

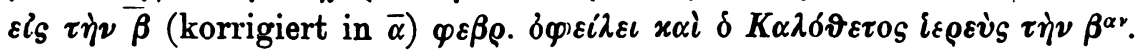

(Diese Linie findet sich genau so in der Hs. Vgl. unten S. 154.)

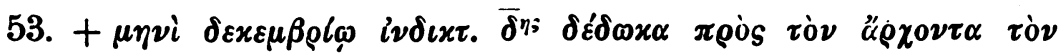

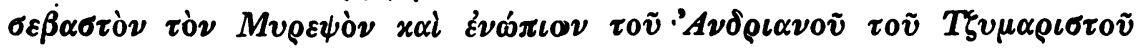

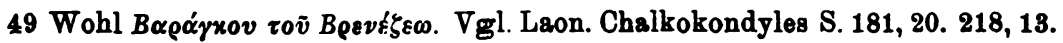




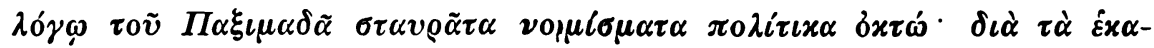

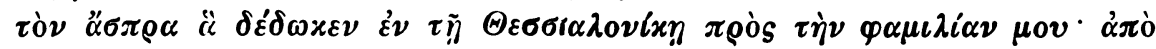

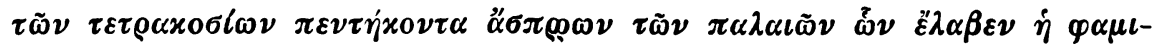

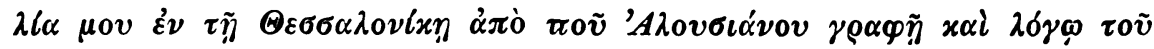

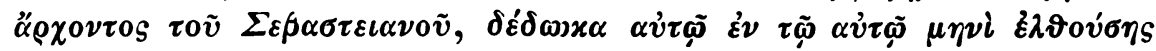

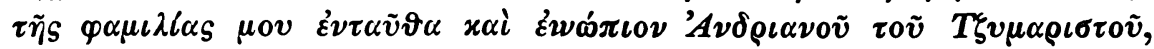

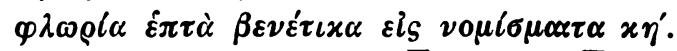

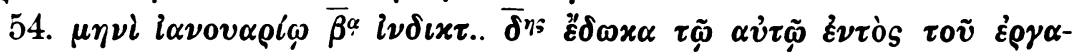

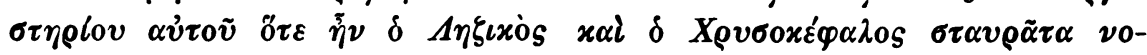
$\mu i \sigma \mu \alpha \tau \alpha \delta$.

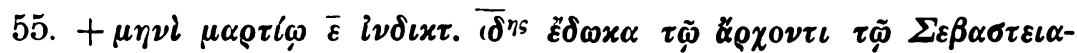

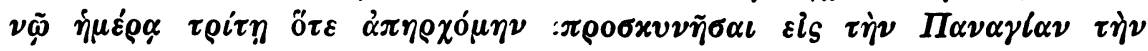

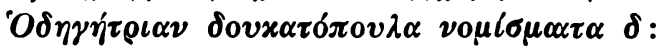

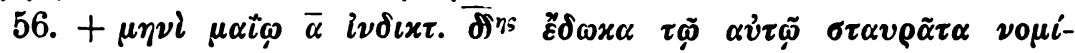
$\sigma \mu \alpha \tau \alpha \delta \dot{v} \mathbf{0}$ :

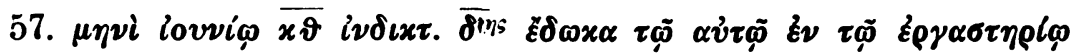

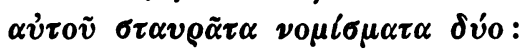

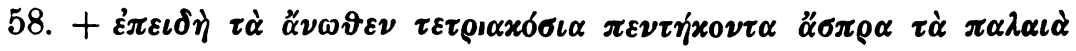

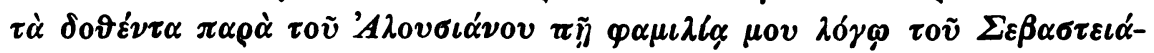

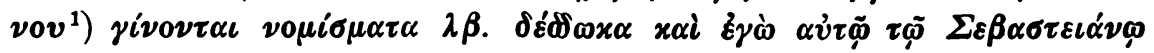

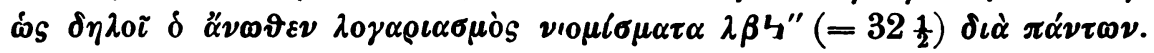

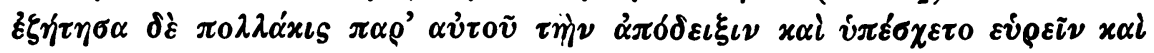

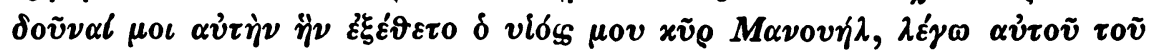

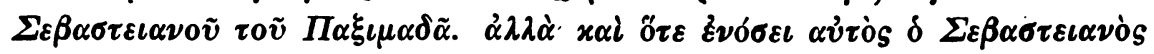

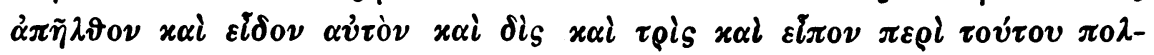

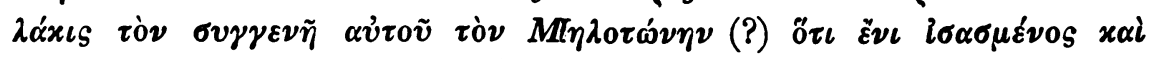

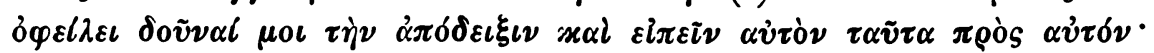

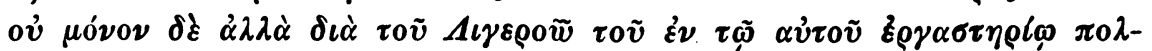

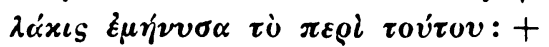

(Das folgende Drittel des f. $2^{v}$ ist leer.)

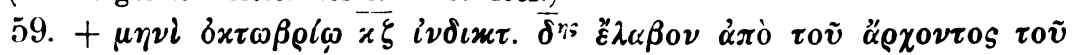

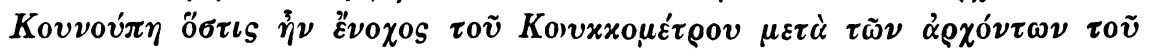

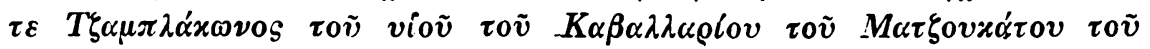

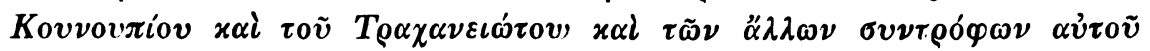

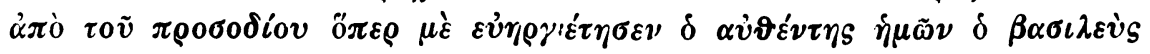

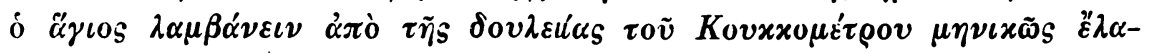

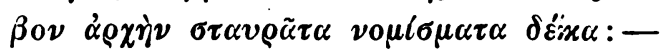

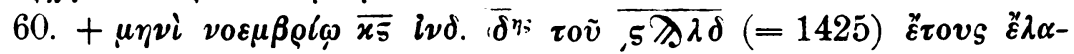

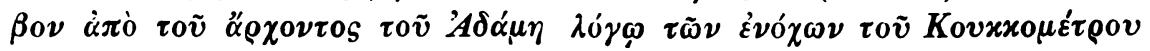

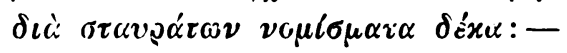

1) korrigiert aus $\sum \varepsilon \beta \alpha \sigma \tau \varepsilon \iota \alpha \nu$ v . 


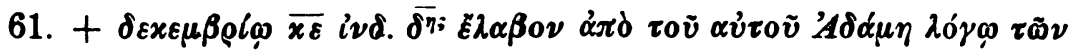

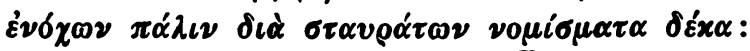

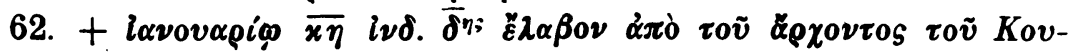

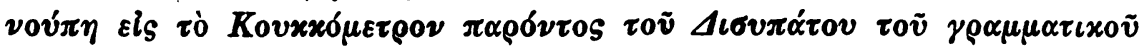

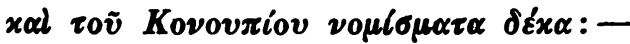

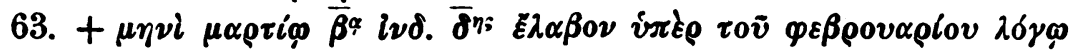

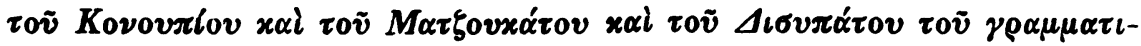

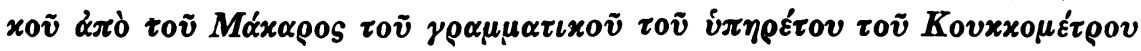

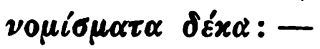

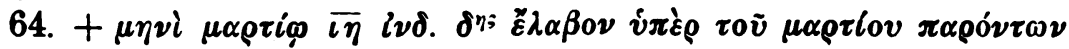

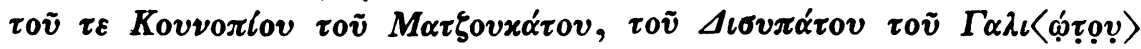

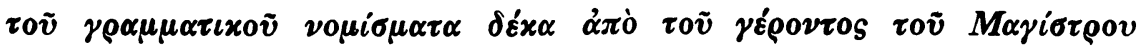

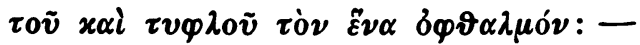

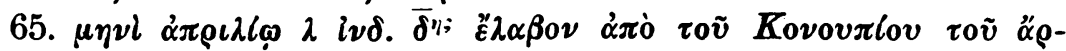

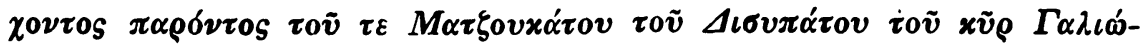

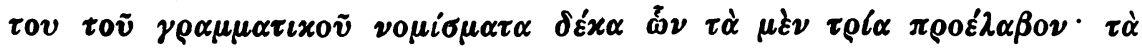

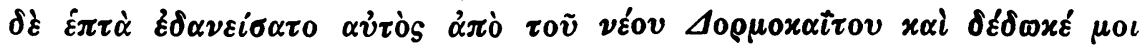
$\tau \alpha \tilde{v} \tau \alpha:-$

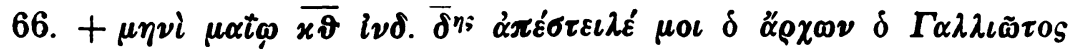

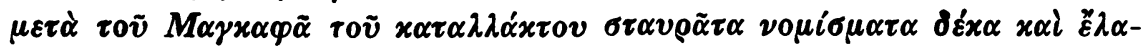

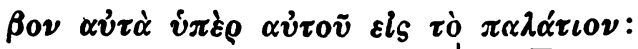

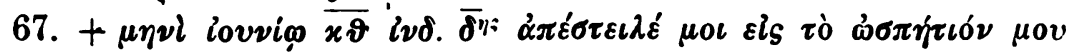

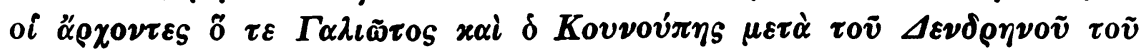

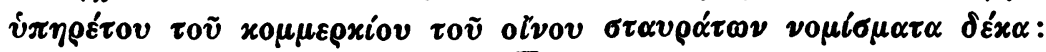

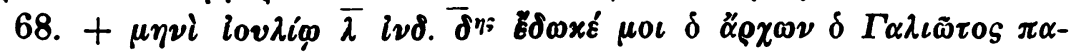

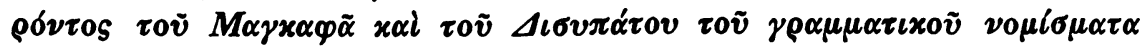
$\delta \varepsilon x \alpha:+$

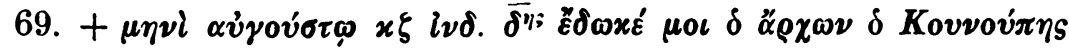

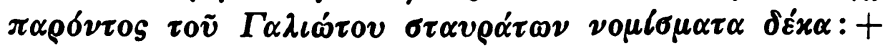

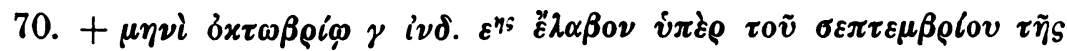

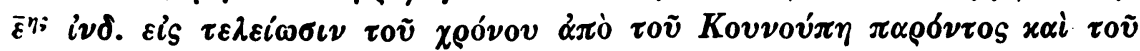

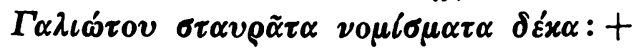

(Die andere Hălfte von f. $3^{r}$ ist leer; ff. $3^{r}$ u. $4^{r}$, welche auch leer gelassen wurden, hat eine spătere Hand mit wertlosen grammatischen Notizen gefüllt.)

f. $4^{\nabla}$ (das obere Drittel leer).

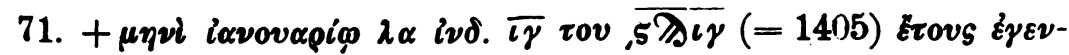

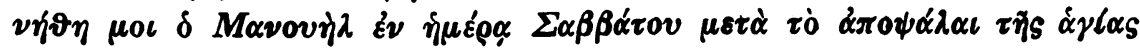

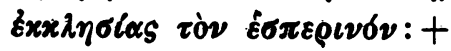

(Der Rost von $f^{4} 4^{*}$ ist leer gebliebon.)

72. f. $5^{\mathrm{r}}+\tau \tilde{\eta} x \xi$ (leerer Platz für 2 Zeilen).

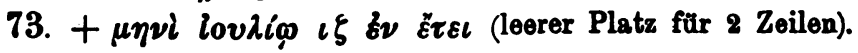




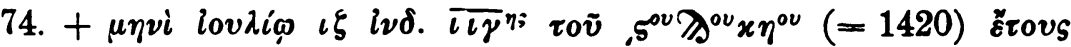

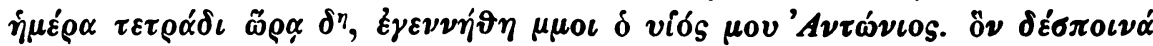

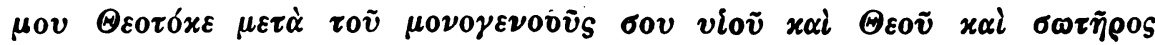

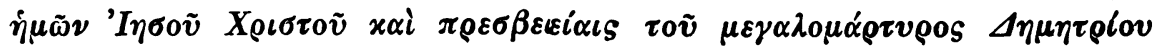

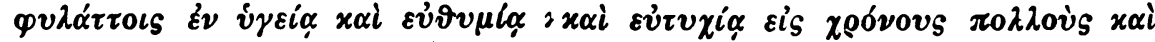

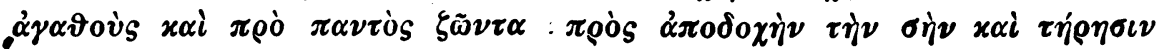

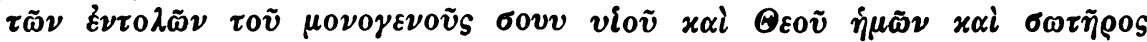

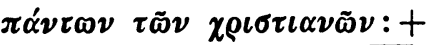

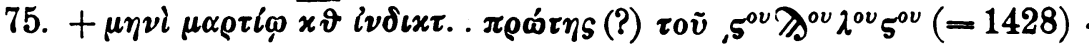

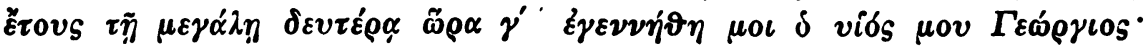
ö $\nu$ X

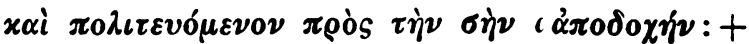

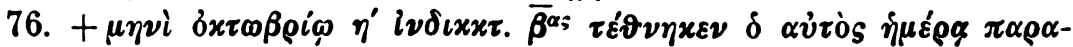

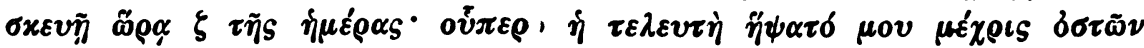

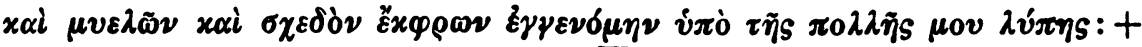

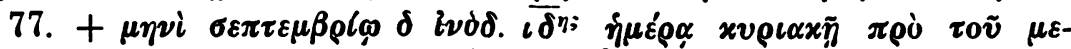

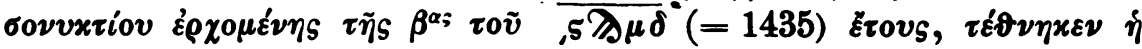

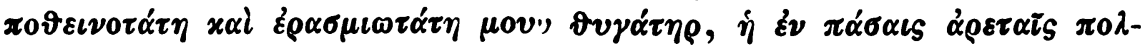

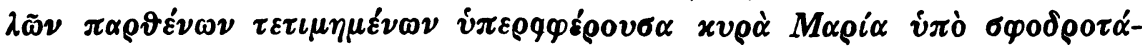

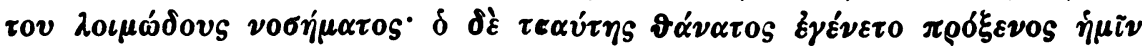

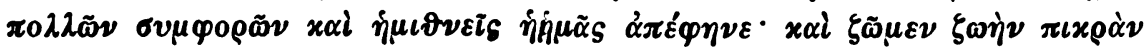

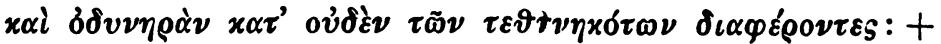

f. $5^{\circ}$ (die erste Hälfte loer).

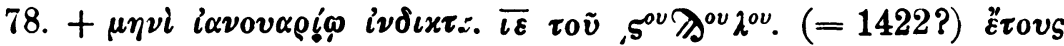

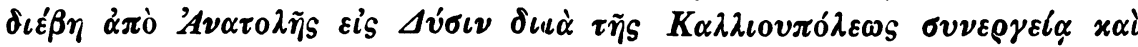

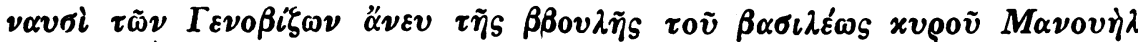

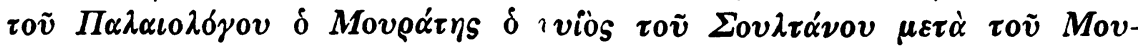

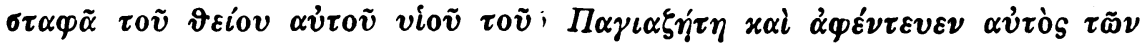

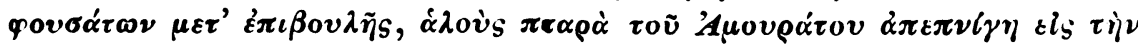

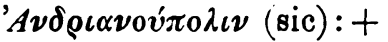

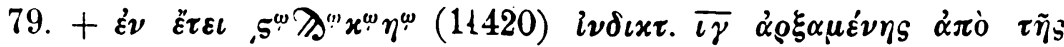

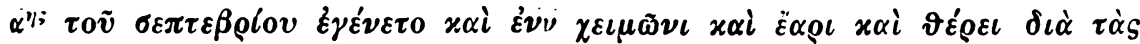

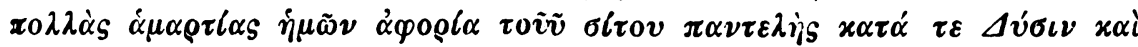

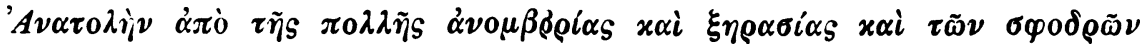

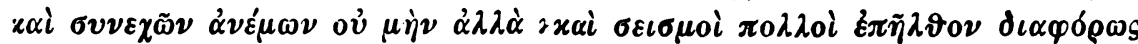

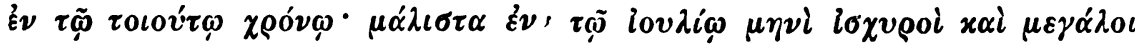

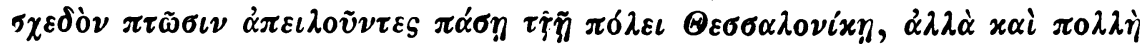

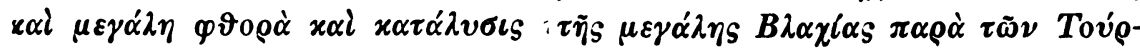

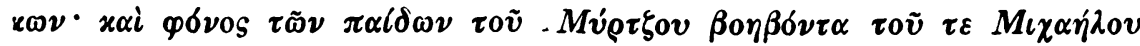

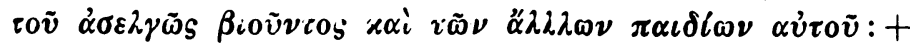

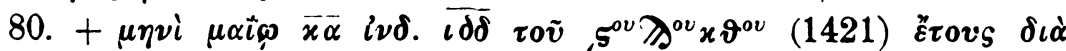




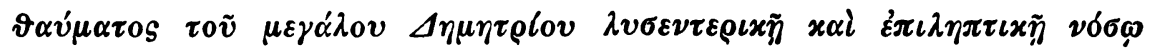

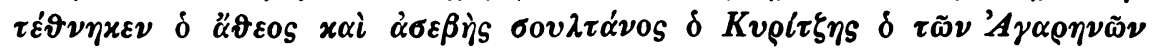

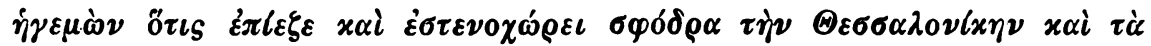
$\hat{\rho} \omega \mu \alpha i x \dot{\alpha} \pi_{\alpha} \nu \tau \alpha \pi \rho \dot{\gamma} \gamma \mu \alpha \tau \alpha$ :

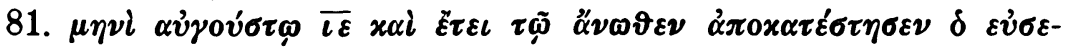

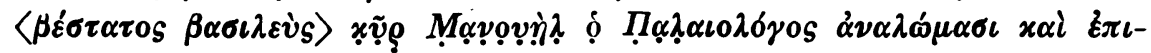

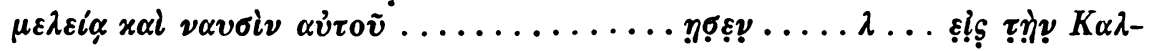

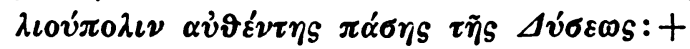

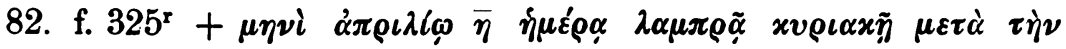

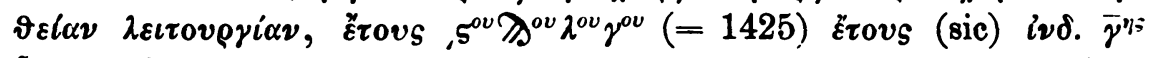

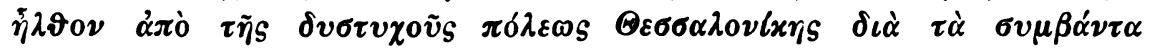

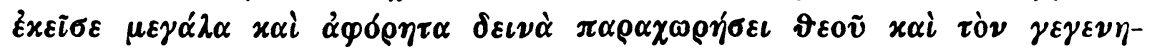

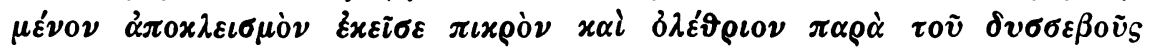

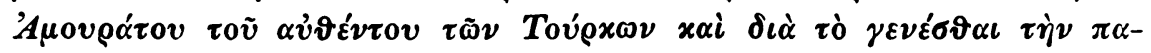

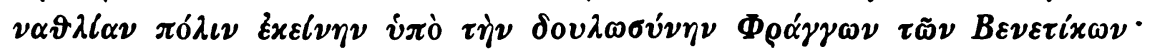

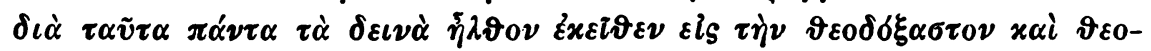

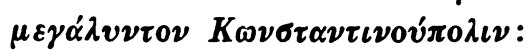

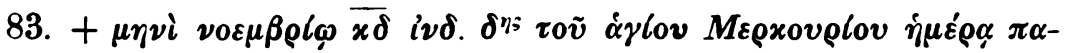

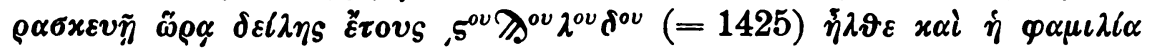

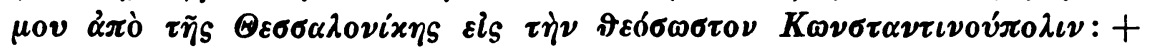

(Es folgt ein leerer Platz, auf den eine spătere Hand folgendes Wortspiel ge-

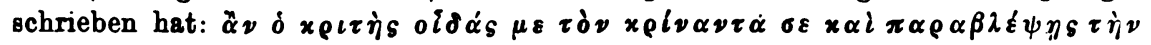

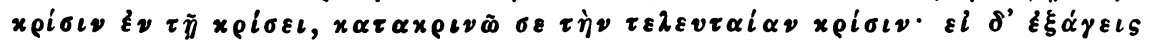

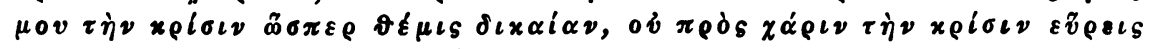
$\mu \varepsilon \Phi \iota \lambda \dot{\alpha} \nu \vartheta \rho \omega \pi \varepsilon x \rho \iota \tau \dot{\alpha} \varepsilon \nu \times \rho l \sigma \varepsilon \iota:)$

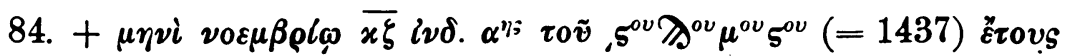

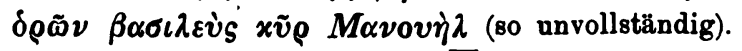

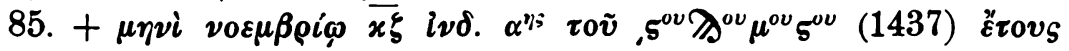

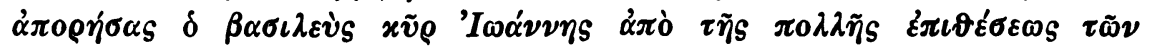

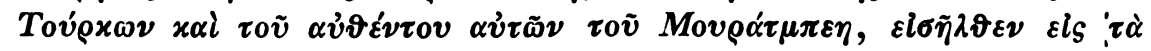

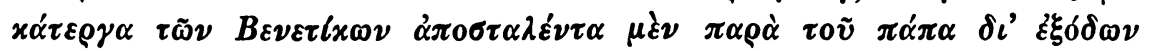

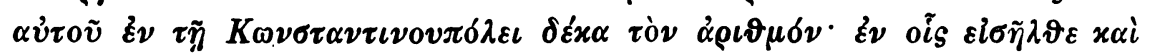

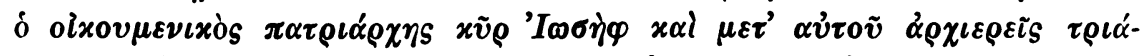

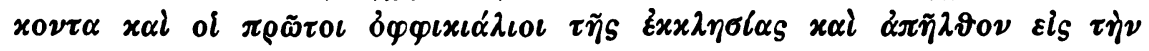

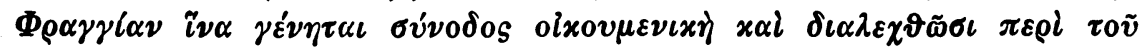

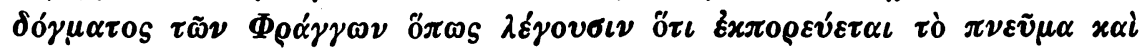

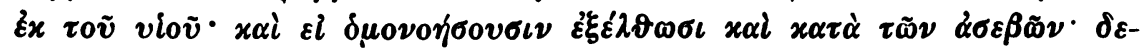

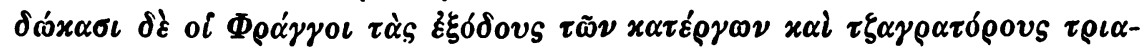

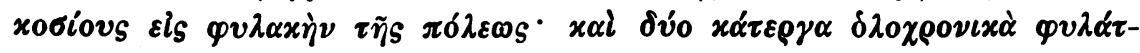

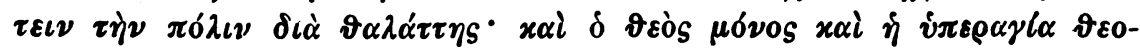

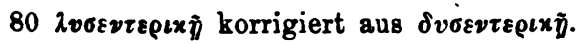




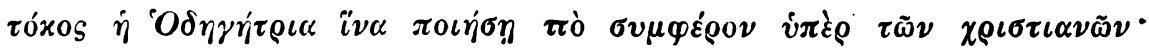

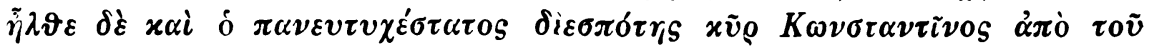

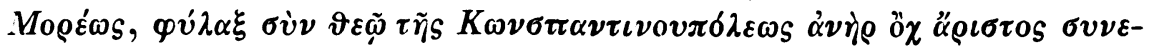

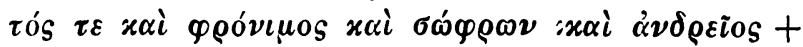

(Es folgt die über Jahre und Wochhen handelnde Notiz, zweimal geschrieben, siehe unten S. $15 j \mathrm{Anm} .^{2}$.)

f. $325^{\text {v }}$.

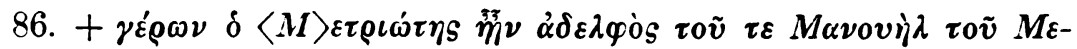

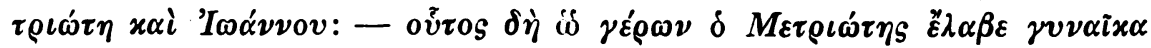

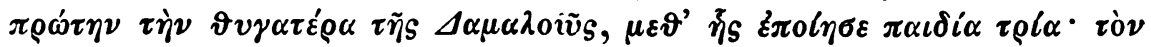

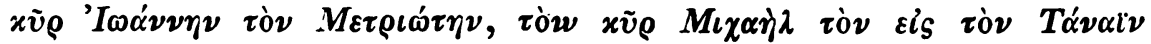

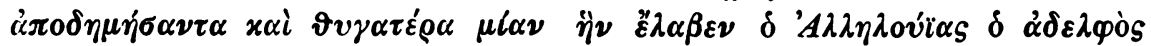

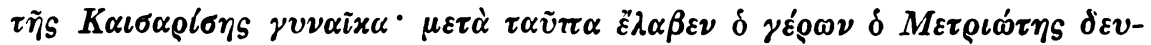

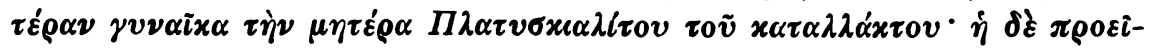

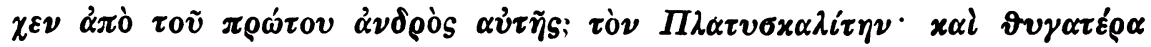

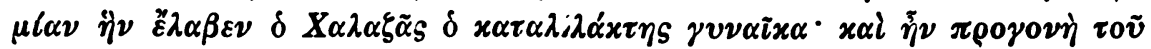

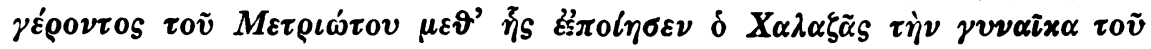

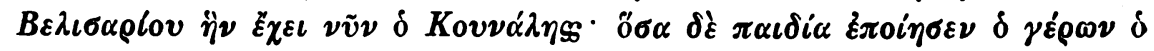

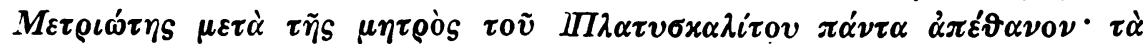

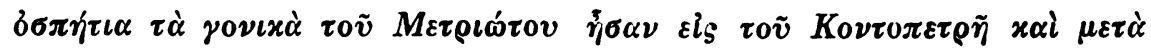

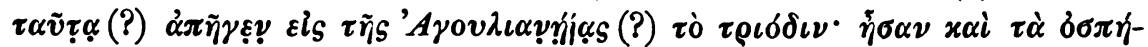

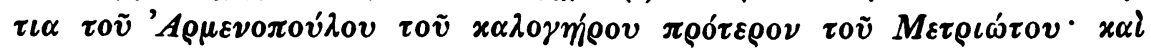

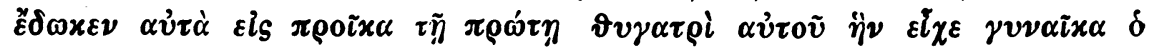

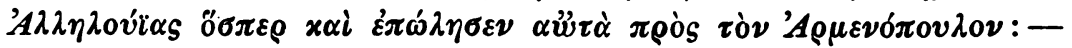

(Das übrige, ebenso wie ff. 326-327 ${ }^{r}$ sind leer geblieben; f. $327^{\circ}$ von späterer

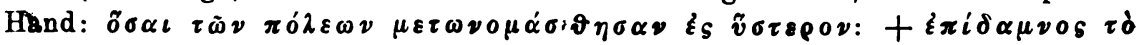
$\nu \tilde{v} \nu \Delta v \rho \rho \alpha ́ x \iota 0 v$ x. $\tau$. $\lambda$. (siehe unten). f. $328^{\mathrm{r}}$ die metrischen Notizen (siehe unten).

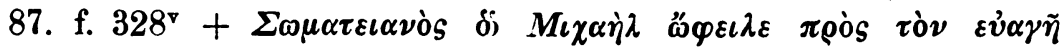

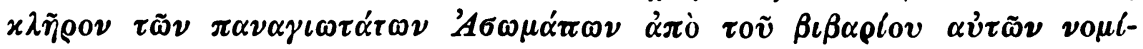

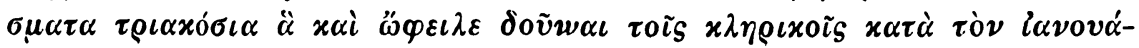

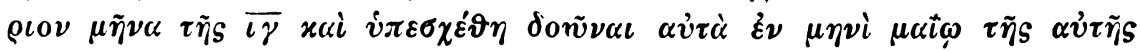

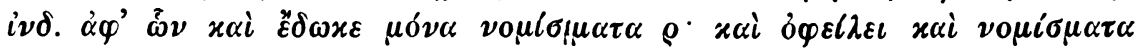

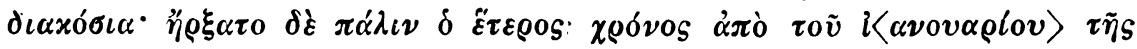

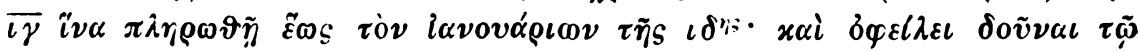

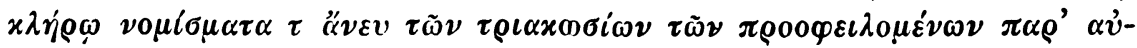

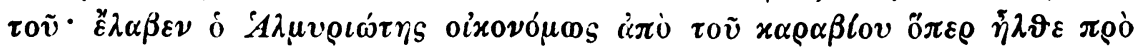

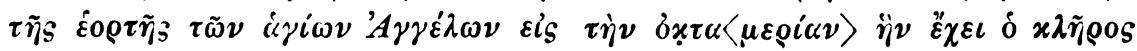

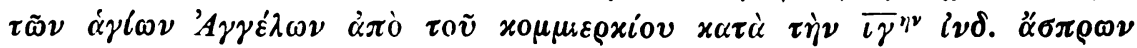

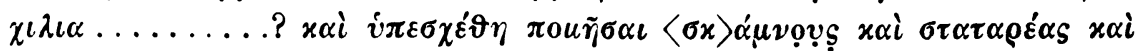

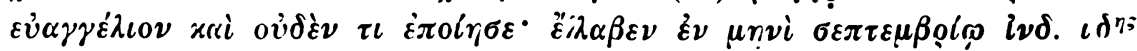

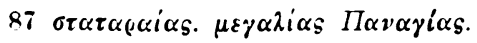




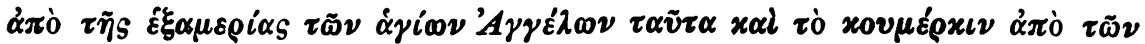

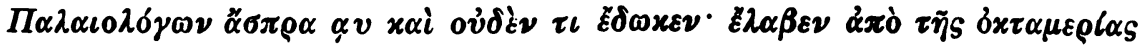

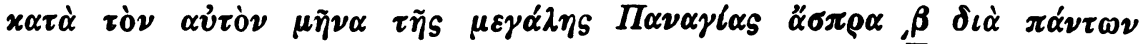

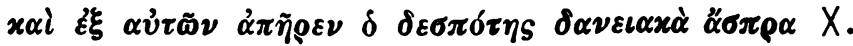

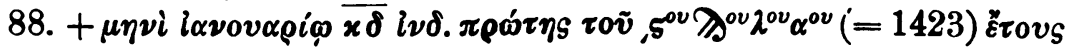

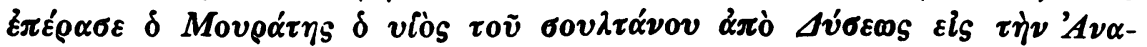

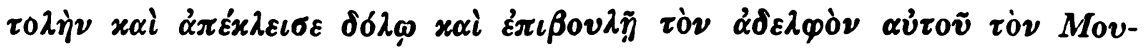

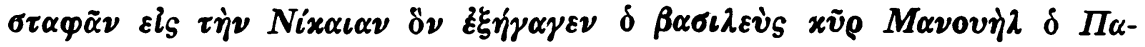

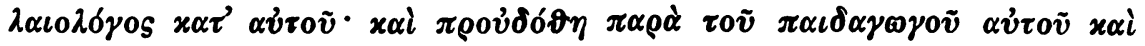

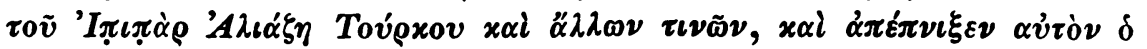

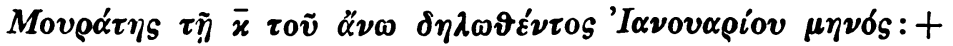

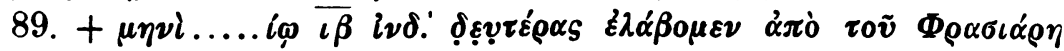

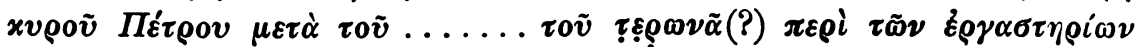

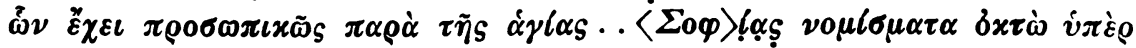

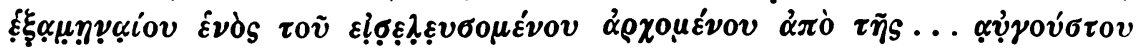

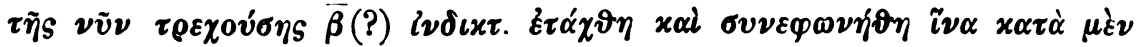

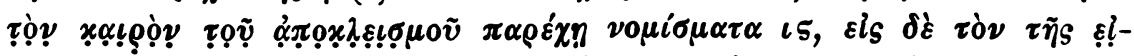

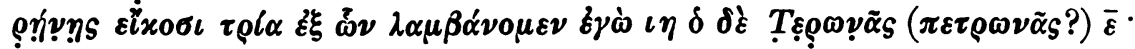

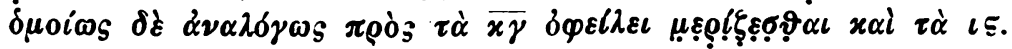

\section{§ 3. Charakteristik.} werden:

Die Notizen können dem Inhalte nach in drei Gruppen zerlegt

I) Wirtschaftsnotizen (Nr. 1-70). Diese stehen auf den ff. $A^{\top}$, $1^{r}, 1^{r}, 2^{r}, 2^{r}$ und $3^{r}$. Die untere Hälfte von f. $3^{r}$ nebst $3^{r}$ und $4^{r}$ hat der Notizenschreiber unbeschrieben gelassen, offenbar zur Fortsetzung seiner Wirtschaftsnotizen. Auf diesen leeren Platz hat eine spätere Hand wertlose Notizen grammatischen Inhalts eingeschrieben. Diese wirtschaftlichen Notizen, in welchen der alte Buchbesitzer angibt, wann, woher, weshalb und wieviel Geld er einkassiert, oder wann, wo, an wen und weswegen er bezahlt hat, sind teils in Thessalonike (Nr. 1 -52), teils in Konstantinopel (53-70) geschrieben. Die beiden Perioden wurden vom Schreiber selbst durch eine wagerechte Linie voneinander getrennt (f. $2^{r}$, siehe oben Notiz Nr. 52). Wirtschaftliche Notizen mit andern geschichtlichen Inhalts sind auch auf $f$. $328^{r}$ enthalten (Nr. 87); hier aber handelt es sich um gemeinsame Rechte und Ansprüche verschiedener Teilhaber. Der alte Besitzer des Aristidesbuches schrieb also seine persönlichen Geschäftsnotizen gesondert von den mit anderen gemeinsamen Rechnungen.

II) Familiennotizen (Nr. 71-77. 82-83). Von diesen findet sich Nr. 71 allein für sich auf dem untersten Teil von $f .4^{\mathbf{r}}$; diese Seite 
hat der Schreiber fast vollständig leer gelassen, da er offenbar beabsichtigte, diese Familiennotizen mit der Zeitangabe seiner Vermählung, vielleicht auch seiner Priesterweihe und sicher der Geburt seiner Tochter Maria zu ergänzen, von der lediglich der Tod in Notiz 77 angegeben wird. Nr. $72-77$ finden sich auf f. $5^{r}$, dessen gröBter Teil wiederum, wie auch die obere Hälfte von $f . \check{o}^{\nabla}$, zur Mitteilung ähnlicher Ereignisse leer gelassen ist.

III) Historische Reminiszenzen (Nr. 49. 78-81. 82. 84. 85. 88). Von diesen sind 49. $78-81$ und 88 in Thessalonike geschrieben und beziehen sich auf diese Stadt, 82-84 auf Konstantinopel.

IV) Juristisch-kanonistische Notizen. Eine solche ist Nr. 86 (f. 325 ${ }^{\vee}$ ), die in Thessalonike geschriebem sich auf eine Erbschaft zwischen

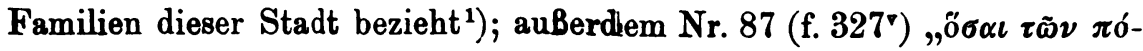

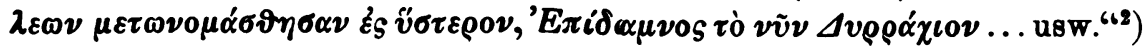

V) Metrische Notizen (f. $\left.325^{x}\right)$ ). In diesen, die von ganz untergeordneter Bedeutung sind, werden dau ,

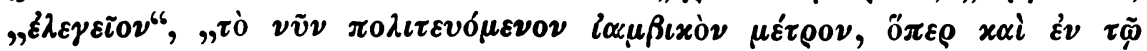

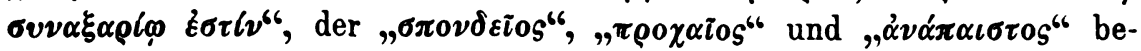
sprochen.

\section{§ 4. Zeitf olge.}

Die Notizen sind zu verschiedenen Zeiten vom Jahre 1419 bis 1438 geschrieben und, wie gesagt, teills in Thessalonike, teils in Konstantinopel. Die chronologische Reihemfolge ist eingehalten: 1419: $\mathrm{Nr}$. 1-8. 10-25. 74. 79. 1420: Nr. 26 -37. 80. 87. $1421:$ Nr. 38-50. 78. 1422: Nr. 9. 51. 88. 1423: Nr. 52. 1424: Nr. 89. 1425: Nr. 82-83. 1426: Nr. 53-69. 1427: Nr. 70. 1428: Nr. 75. 1433: Nr. 76. 1435: Nr. 77. 1437: Nr. 84-85. Nr. 71 ist nicht im Jahre 1405, dem Geburtsjahr des Sohnes Manuel, sondern später und, wie es scheint, aus dem Gedächtnis geschrieben. Aus dem auf f. $4^{v}$ leer gelassenen Platz läBt sich schlieBen, daB der Buchbesitzer beabsichtigte, noch andere

1) Als thessalonikisch sind die in dieser Notiz vorkommenden Familiennamen $\Pi \lambda \alpha \tau v \sigma x \alpha \lambda i \tau \eta_{S}(V i z$. Vrem. Bd. 13 Beilage XLIV, 18. Ebenda Bd. 17, 106, 3) Kov-

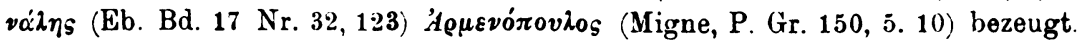

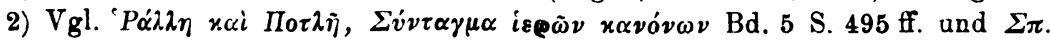

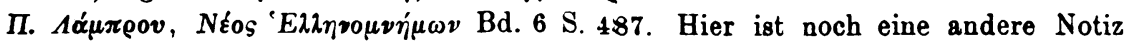

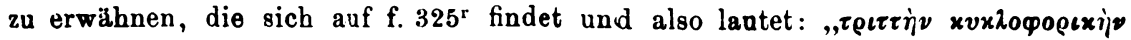

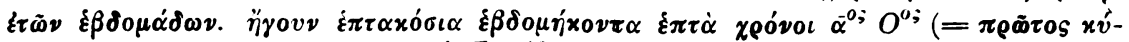

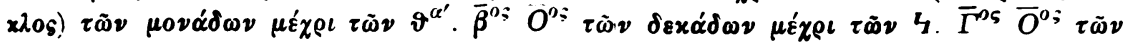

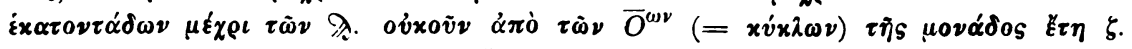

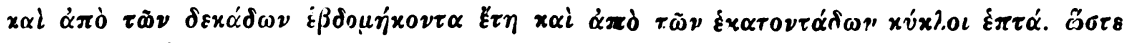

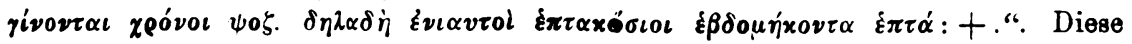
Notiz scheint aber von einer anderen Hand zu stammen. 
Familiennachrichten einzutragen. Die zwischen Nr. 8 und 10 bestehende ? Unordnung in der Zeitfolge ist damit zu erklären, daB der Notizen- schreiber die Notiz 9 aus Mangel an Platz auf dem untersten Rand 1 von f. $A^{\top}$ geschrieben hat.

\section{§ 5. Inhaltsanalyse.}

Eine ausführliche Analyse des oben mitgeteilten Textes, in welchem । die Nachrichten so klar für sich selbst sprechen, erscheint überflüssig. . Der Schreiber notiert die Summen, die er aus dem Einkommen der sechs Hauptkirchen in Thessalonike, der des Heiligen Demetrios (Nr. 6. . 15. 16. 20. 27. 39. 40. 42. 45), der Heiligen Sophia (Nr. 5. 12. 17. 22. 27. . 41. 88), der Heiligen Asomatoi (Nr. 3. 18. 23. 28. 31. 32. 34. 38. 44. 86), , des Heiligen Menas (Nr. 7. 8. 19. 21. 35. 47. 51), der Acheiropoietos' (Nr. 4. 10. 28. 30. 32. 38. 50. 52) und der Hodegetria (Nr. 33. 43. 51.. כ̄ā), bezog oder zu beziehen berechtigt war. Dieses Einkommen aus Festen 1

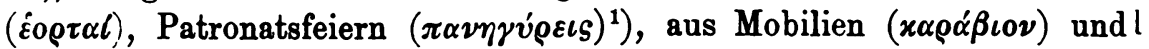

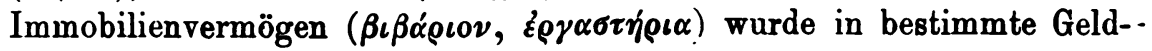
summen und Naturalbeträge (17. 40) zerlegt, die am SchluB des Se-mesters oder der Indiktion (10.12) unter die verschiedenen Berechtigten । verteilt wurden (37). Manchmal verweigern die Kirchenbeamten die Aus--

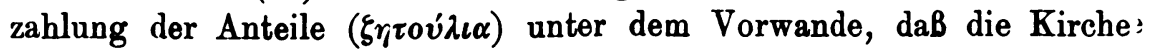
verschiedene Gebrauchsgegenstände nötig habe (31. 87).

Außer dem Einkommen aus den Kirchen hatte der Notizenschreiber: auch das Recht, einen Teil von der Miete einiger Werkstätten zu er-heben (13. 14. 24. 25. 26. 46. 48). Diese Werkstätten ( $\mu v \rho \varepsilon \psi \iota x \alpha \grave{~ z ́ \rho \alpha--~}$

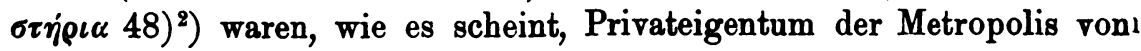
Thessalonike, da über sie von dem damaligen Metropoliten Symeonı verfügt wurde (26. 46).

Die Geldanteile entrichteten die Priester der verschiedenen Kirchen,

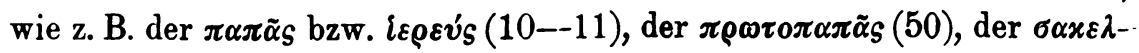

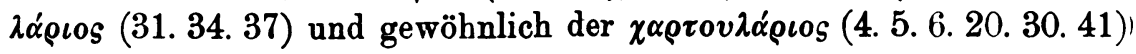
entweder direkt dem Notizenschreiber oder indirekt durch einen dritten, z. B. durch seinen Bruder (21. 33. 37), häufiger aber durch einen $\mathrm{Me-}$

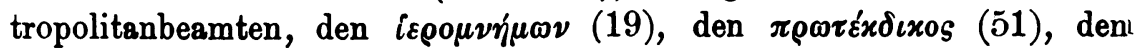
$\sigma \alpha x \chi \varepsilon \lambda$ lov (35. 36), den $\chi \alpha \rho \tau o \varphi v ́ \lambda \alpha \xi$ (6. 42). Letzterer schlieBt manch-

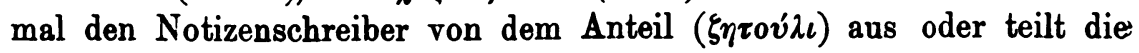

1) Ubber das Fest $\Delta \eta \mu r i r \rho \alpha$ des Heiligen Demetrios rgl. Tafrali, Thessalonique au XIVe siecle (Paris 1913) S. 138 ff. und die dort angeführte Literatur.

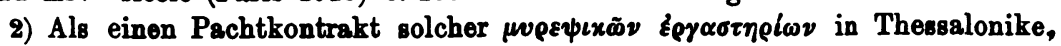
wo auch der in Notiz 1 erwahnte Namo $\Sigma \alpha \mu \alpha \mu l \nu 0 \eta s$ wiederkehrt, vgl. die Urkunde rom Jahre 1400 bei Miklosich-Müller, Acta et Diplomata II, S. 625. 


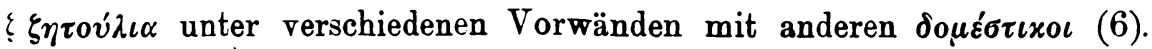
- Aus allen diesen Notizen, besonders aber aus der Notiz 6, wo von den ( $\left.\delta \circ \mu \varepsilon^{\prime} \sigma \tau \iota x \iota^{1}\right)$ als Mitberechtigten die Rede ist, und aus der Einmischung 1 des Metropoliten in die Auszahlung des Einkommens (26. 42), geht l klar hervor, daB unser Notizenschreiber zu dem Personal der Metro( polis gehörte mit Anrechten auf Teile des Metropoliseinkommens. ${ }^{2}$ )

Die Notizen sind vielfach interessant und lehrreich. Erst jetzt werden wir davon unterrichtet, wie die Metropolisbeamten ihr Gehalt bekamen. ${ }^{3}$ ) Die Metropolis von Thessalonike erhob neben dem Einkommen aus ihren Privatbesitztümern ${ }^{4}$ ) noch Beihilfsgelder von den Hauptkirchen der Stadt und besoldete damit ihre Beamten. Das Kirchenc einkommen wurde in so viele Teile geteilt als Anwärter vorhanden waren. Die Zahl von Anteilen und Teilnehmern bei jeder Kirche stellte wahrscheinlich der Metropolit fest; das ist der Grund dafür, daB die Metropolis von Thessalonike auch in früheren Zeiten Klöstergüter als Kirchengüter zu charakterisieren versuchte, um auch davon den diesbezüglichen Anteil zu beanspruchen. ${ }^{5}$ )

Auch für die Sozialverhältnisse der Stadt Thessalonike sind die Angaben des Notizenschreibers lehrreich. Die Notizen 1 und 2 bieten interessante Nachrichten über Sklavenverkauf von türkischer Seite an Christen. ${ }^{6}$ ) Notiz 9 spricht von einer gewissen $P \alpha \delta \eta \nu \eta^{7}$ ), die Geld auf Pfand leiht.

1) Vgl. Philothei, Encomium Gregorii Palamae bei Migne P. Gr. Bd. 151, S. 638 D:

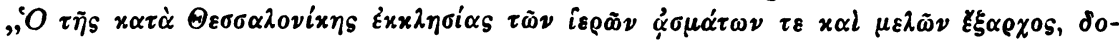

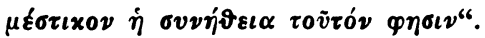

2) Leider besitzen wir aus jener Zeit keine Urkunde der Metropolis von Thessalonike, um die Unterschriften der verschiedenen Officialen kontrollieren zu können. Vgl. z. B. die aus dem Jahre 1371 stammende Thessalonikeurkunde (Mi-

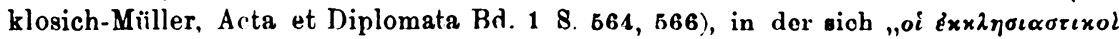

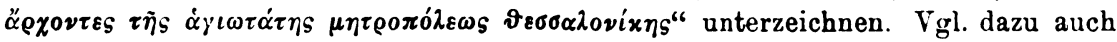
Actes de l'Athos in Vizant. Vremennik, Beilage des 17. Bd. Nr. 6-12).

3) Tafrali, den ich auf diesen für die Thessalonikekirche hochinteressanten Text aufmerksam machte, vermochte diese Notizen nicht genügend zu benutzen, obwohl er sie in seinem Buche wiederholt zitiert.

4) Utber diese kirchlichen Besitztümer im allgemeiuen vgl. 'Tafrali, a. a. O. S. 111 Anm. 5.

5) Vgl. Miklosich-Müller, Acta et Diplomata Bd. I S. 191, wo die Mönche des Asomatoiklosters im Jahre 1339 mit dem Metropoliten streiten und Bd. Il S. 519, wo der Patriarch von Konstantinopel dem Metropoliten von Thessalonike Vorwürfe

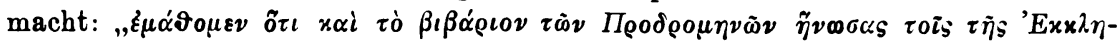

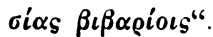

6) Utber den von den Türken geübten Sklavenhandel vgl. Tafrali, 3. 3. 9. S. $38 \mathrm{ff}$.

7) Dieser byzantinische Familienname P $\alpha \delta \eta v o ́ s$ ist schon bekannt. Vgl. 7. B. 
Die in Konstantinopel geschriebenen Notizen sind nicht minder · interessant. Als unser Notizenschreiber Thessalonike verlassen hatte,

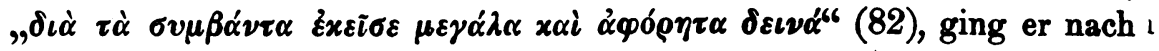

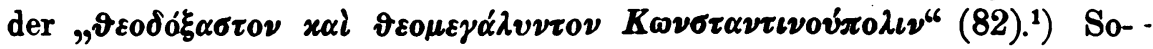
bald er in die Hauptstadt gekommen war, kam er unter die hohe Pro- tektion des damaligen Kaisers Joannes Palaiologos, auf dessen Befehl l er ein monatliches Gehalt ( $\pi \rho \circ \sigma o ́ \delta \iota 0 \nu)$ aus dem Einkommen des Kov- .

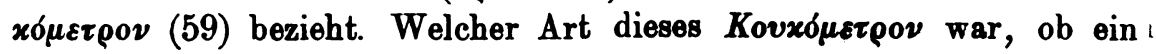
königliches Gut oder ein $x о \mu \mu \varepsilon^{\prime}\left(x \nu^{2} \nu^{2}\right.$ ), ist nicht sicher zu sagen; wahrscheinlich scheint mir, daB es sich um ein kaiserliches Gut handelte..

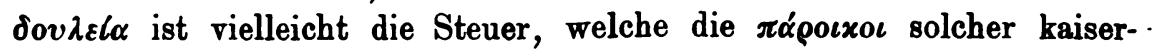

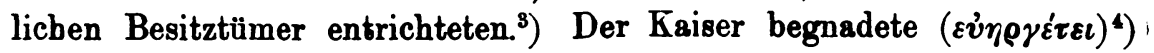
unter den verschiedenen $\ddot{\alpha} \varrho \chi 0 \nu \tau \varepsilon \xi$ einige als zur Erhebung dieser Steuer berechtigt (

Nach den Notizen ist anzunehmen, daB das Einkommen aus dieser

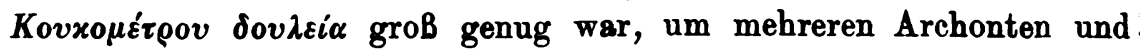
edlen Byzantinern, deren Namen in den Notizen 59-70 angeführt sind,, Anteile zu gewähren. Der Schreiber notiert, daß er innerhalb eines Jahres, vom Oktober 1425 bis Nojember 1426, eine monatliche Zulage von zehn $\sigma \tau \alpha v \rho \alpha \tilde{\tau} \alpha$ erhielt. Die mit dem, so viel ich weiß, sonst nicht

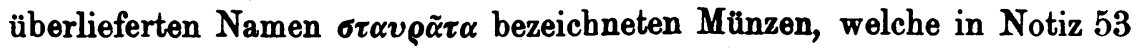
auch $\pi 0 \lambda l \tau \iota x \alpha$ genannt werden, sind jedenfalls die mit einem Kreuz

Viz. Vrem. Bd. 17 (Beilage) Nr. 66, 6, 13. 73, 5. Schlumberger, Sigillographie de l'empire Byzantin S. 573. 694.

1) Uber die in jener Zeit erfolgte Utbersiedelung von Thessalonikern nach der Hauptstadt vgl. Tafrali, a. \&. O. S. 16.

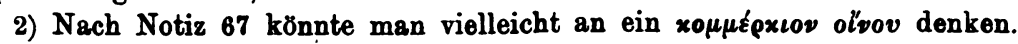

3) Vgl. x. B. Aotos de l'Athos in Virant. Vromennik, Bd. 17 Beilege 58, 22

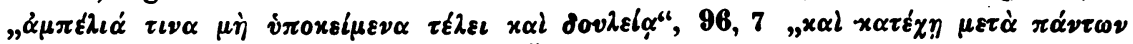

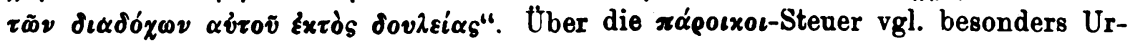
kunde 92 v. $18 \mathrm{ff}$.

4) Vgl. Sgouropoulos, Historia concilii Florentini Hagae $1660 B^{\prime}, x:$ : Evvx

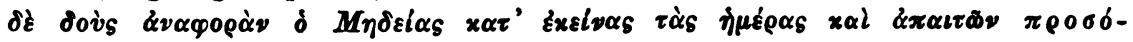

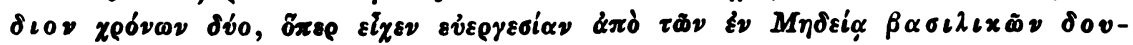

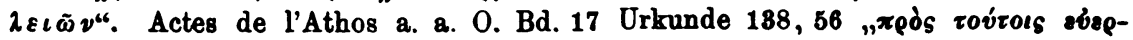

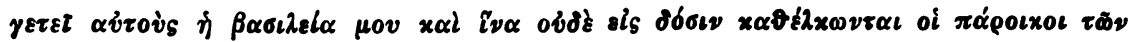

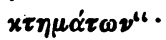

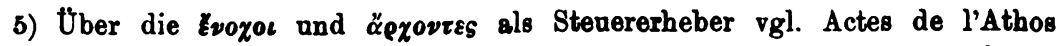

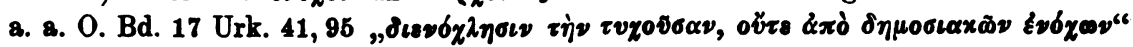

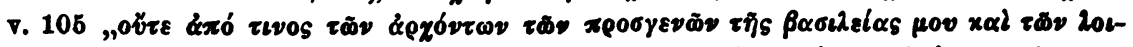

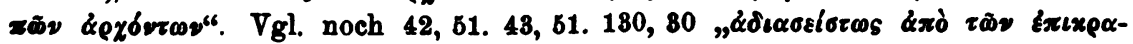

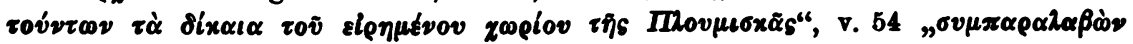

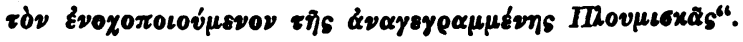


versehenen Paläologenmünzen ${ }^{1}$ ) und, wie aus der Notiz hervorgeht, war ihr Wert ungefähr $12^{1} / 2$ Aspra.

Aber auch im allgemeinen vervollständigen diese wirtschaftlichen Notizen unsere Kenntnisse des byzantinischen Münzwesens. So wird z. B. durch Notiz 4 neuerdings bestätigt, daB das Hyperpyron kein Geldstück, sondern eine Geldsumme war. In den Notizen 4 und 7 werden die $\chi \alpha \lambda x \tilde{\alpha}, \pi \alpha \lambda \alpha \iota \alpha^{\prime}$ und $\dot{\varepsilon} \lambda \omega \varphi \rho \dot{\alpha} \ddot{\alpha} \sigma \pi \rho \alpha$ von den $\dot{\alpha} \rho \gamma v \rho \tilde{\alpha}$ unterschieden. $\left.{ }^{2}\right)$

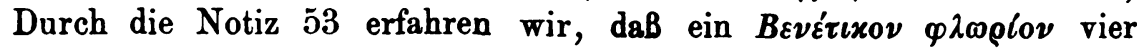
Hyperpyra galt. Hochinteressant, jedoch nicht ganz klar, ist auch Notiz 58 in bezug auf das Verhältnis zwischen den verschiedenen byzantinischen Münzen.

Auch die byzantinische Onomatologie wird durch diese Notizen bereichert, da man unter den angeführten Familien- und Ortsnamen einigen hier zum erstenmal begegnet.

Endlich sind die Notizen, die ein Gelehrter, aber ganz für sich, in halbvulgärer Sprache geschrieben hat, auch sprachlich nicht ohne Interesse. Unter den seltenen Worten und Typen, wie z. B. $\delta \alpha \nu \varepsilon \iota \alpha x \alpha^{\prime}, \xi_{\xi} \alpha-$ $\left.\mu \varepsilon \rho i \alpha, \sigma \tau \alpha \tau \alpha \rho \varepsilon^{3} \alpha^{3}\right)$, finden sich einige, die in die gewöhnlichen Lexika

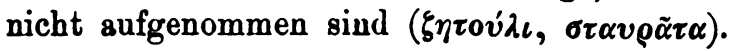

Die geschichtlichen Reminiszenzen treffen meistens bekannte Tatsachen ${ }^{*}$; die Familiennotizen lassen den Schreiber als einen frommen, der Kirche ergebenen und seine Kinder zärtlich liebenden Mann erkennen. Eine besondere Aufmerksamkeit erweckt Notiz 9, die von der schlechten finanziellen Lage des Schreibers spricht, die ihn gezwungen

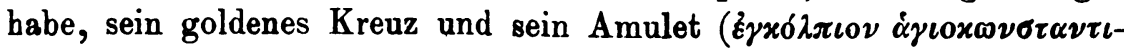

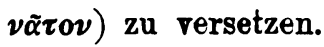

\section{§6. Der Schreiber.}

Wer unser Notizenschreiber war, können wir leider nicht sicher sagen, da er nirgends seinen Namen angibt. Mehrere Indizien sprechen für Joannes Eugenikos. Nicht nur die Zeit der Notizen, sondern auch die Interessen (kanonische, metrische), die sie bekunden, passen gut zu dem, was wir von Joannes Eugenikos wissen. Dieser war Diakon und Nomophylax, hat metrische Versuche, Hymnen, Epigramme, Rätsel usw.,

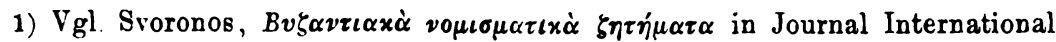
d'Archéologie Numismatique Bd. 2 S. 364.

2) Ebenda S. $348 \mathrm{ff}$.

3) Das Wort findet sich nur bei Porphyrogennetos, De Cerimoniis Aulae Byzant. II S. 645. $769 \mathrm{ff}$.

4) Zu den Notizen 49. 78 79. 82 rgl. Phrantzes l. I cap. $31.38 \mathrm{ff}$. Doukas cap. XXIX nnd besonders Laon. Chalkokondyles SS. $79 \mathrm{ff} .227 .235,10 \mathrm{ff}$. Die Daten stimmen freilich nicht. 
Monodien und Ekphrasen geschrieben, was an den rhetorischen Inhalt der ganzen Hs gemahnt. $\mathrm{DaB}$ er das Synodikon der Kirche von Thessalonike ${ }^{1}$ ) und Monodien auf Thessalonikes Eroberung ${ }^{2}$ ) verfaBt hat, ist ein Beweis seiner nahen Beziehungen zu der Stadt und der Kirche. Nach Thessalonikes Fall sehen wir den Joannes Eugenikos in Konstantinopel tätig, und seine verschiedenen Reden an das Paläologenhaus ${ }^{3}$ ) bezeugen ihn als der kaiserlichen Familie ergeben, was durch die in Notiz 59 erzählte kaiserliche Wohltat gerechtfertigt würde. Und da Joannes Eugenikos als Bücherkäufer bezeugt ist ${ }^{4}$ ), könnte er auch der Besitzer unseres Aristideskodex sein. Den Tod der Tochter unseres No-

1) Dieses $\Sigma v v o \delta$ เxóv ist teilweise von L. Allatius, De Symeonum scriptis Diatriba, Parisiis 1664, S. 186 veröffentlicht worden. Aber durch einen Druckfehler, der auch von Migne P. Gr. Bd. 155, S. $10 \mathrm{ff}$. übernommen wurde, steht im Titel Eugenius Nomophylax statt Eugenicus und dementsprechend haben alle, die über dies Synodikon gesprochen haben, einen Eugenius als Verfasser angenommen (vgl. Krumbacher, G. B. L', S. 175-176; Griech. Utbersetzung von Soteriades Bd. $A^{\prime}$, S. 351. Lampros, Neos Hellenomnemon Bd. 9, S. 347) - mit einziger Ausnahme von L. Petit, der (Échos d'Orient Bd. V 1902, S. 96) den Verfasser dieses Synodikons mit dem richtigen Namen Eugenikos zitiert. Weiter unten steht wiederholt sowohl bei Allatius (S. 187-188), wie auch bei Migne (S. 11-14) richtig Eugenicus gedruckt.

2) Vgl. Lampros, N. Hellen. Bd. 5, S. 369. In seiner Monodie auf Konstantinopel (ebenda Bd. 5, S. 323) erinnert er an Thessalonike. Eine Monodie auf die Eroberung von Thessalonike hat auch der Bruder des Joannes, der berühmte

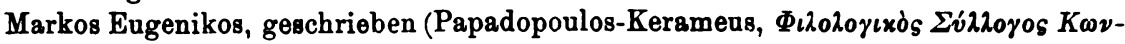

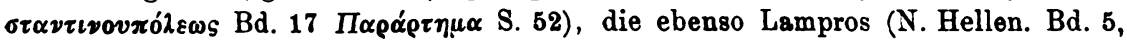

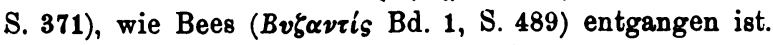

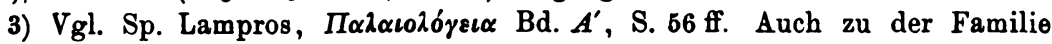
$T \zeta \alpha \mu \pi \lambda \alpha^{\prime} x$ wos, welchen Namen unser Notizenschreiber in Notiz 59 erwähnt, hatten

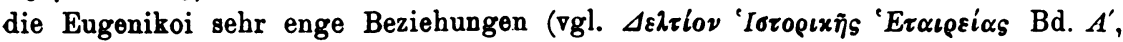

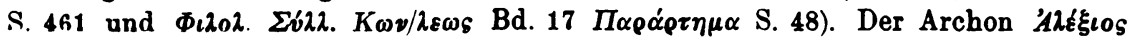

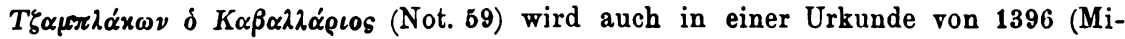
klosich-Müller, Bd. II S. 566) erwähnt.

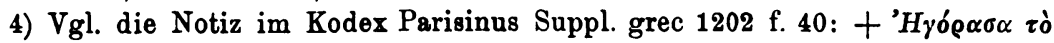

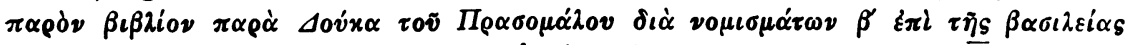

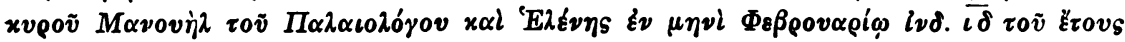
$.5 \lambda_{x} \vartheta \varepsilon_{\text {zovs }}+(1421)$. Die Erwerbung dieses Buches fält mit einem für unseren Notizenschreiber finanziell glücklicheren Jahre zusammen. Sollte der hier genannte

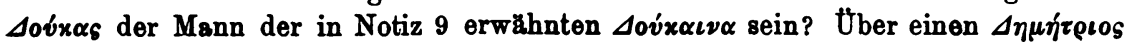

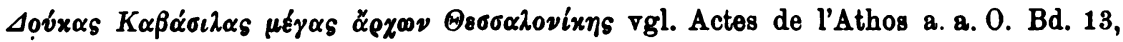

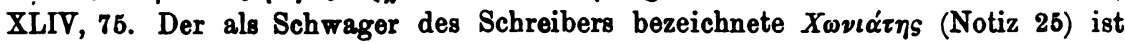

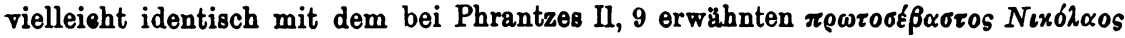

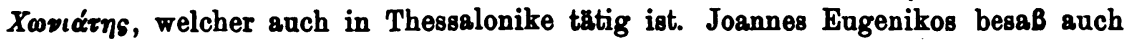
den Kodex Vaticanns Graocus 507 (vgl. Vogel-Gardthansen, Die griechischen

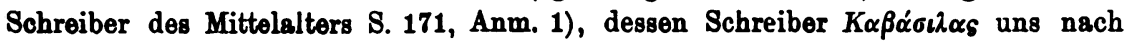
Theseslonike führt, wo diese Familie lebte. 
1 tizenschreibers an der Pest (Nr. 77) könnte man in dem an Sarapion gerichteten Brief des Joannes Eugenikos als angedeutet erkennen. ${ }^{1}$ ) Auch die Namen der Kinder unseres Notizenschreibers sprechen für . Joannes Eugenikos. In den Notizen wird nämlich ein Bruder des Schreibers namens Antonios wiederholt erwähnt (Nr. 1. 21), und diesen Namen seines Onkels erhält auch der 1420 geborene zweite Sobn des Schreibers (Nr. 74). SchlieBen wir nun daraus, daB der Notizenschreiber seinen Kindern Namen gab, die nahen Verwandten angehörten, so stehen wir vor einer Tatsache, die nicht rein zufällig zu sein scheint: die Namen, welche die anderen drei Kinder unseres Notizenschreibers tragen, Manuel (Nr. 71), Georgios (Nr. 75) und Maria (Nr. 77), sind auch Namen von Verwandten des Joannes Eugenikos; Manuel hieB sein Bruder Markos, bevor er zum Geistlichen wurde ${ }^{2}$ ), Georgios und Maria hieBen die Eltern des Eugenikos. ${ }^{3}$ )

Gegen diese Hypothese sprechen aber zwei nicht ungewichtige Gründe. Zunächst hat die Schrift dieser Notizen, wie mir Henri 0 mont freundlich mitteilt, keine sichere Ähnlichkeit mit den als Autograph des Joannes Eugenikos überlieferten Notizen des Codex Paris. suppl. gr. 12024) und des Codex Parisinus gr. $2075^{5}$ ), andererseits steht in

1) Lampros, $\Pi \alpha \lambda \alpha \iota \iota^{\prime} \delta \gamma_{8 \iota \alpha}$ Bd. $A^{\prime}$, S. 162.

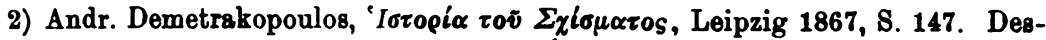

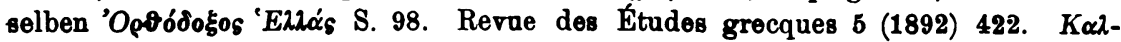

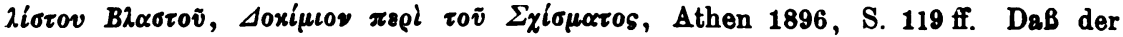
Name Manuel seit alter Zeit in der Familie Eugenikos üblich war, beweist die Urkunde bei Miklosich-Müller, Acta et Dipl. Bd. 2, S. 427, in der der Vater des Joannes Georgios eine Erbschaft von einem alteren Manuel Eugenikos fordert. Dieser Sohn des Joannes Engenikos, Manuel, ist vielleicht identisch mit dem aus dem XV. Jahrh. als Schreiber und Besitzer von verschiedenen Büchern bezeichneten

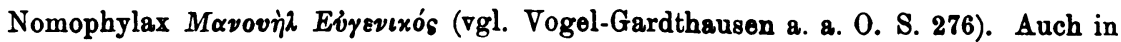
späteren Zeiten (XVI. Jahrh.) finden wir einen Manuel Eugenikos, vgl. Richard

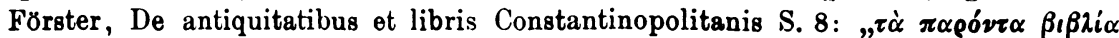

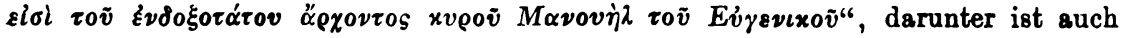
eine Aristideshs (33).

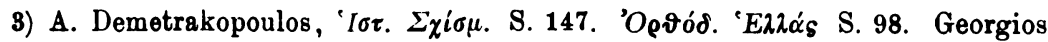

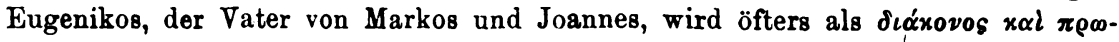

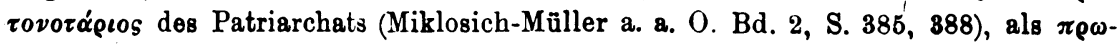

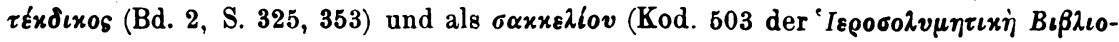
จ auch in Thessalonike tätig und hat dort vom Metropoliten Isidoros die kirchliche

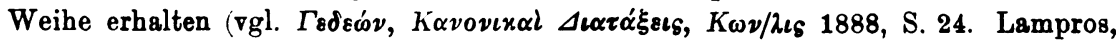

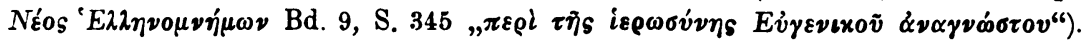

4) H. Omont, Catal. des manuscr. grecs recueillis par Emm. Miller, Paris 1897, S. 62. Vgl. oben S. 160 Anm. 4.

5) Revue des Et. gr. Bd. 5, S. 422. H. Omont, Revue des biblioth. Bd. 2 (1891) S. 10.

Byzant. Zeitschrift XXIII 1 u. 2. 
Notiz 85, wo die Rede ist von den nach Italien abgereisten Synodalgesandten, $\dot{\alpha} \pi \tilde{\eta} \lambda \vartheta o \nu$ statt $\dot{\alpha} \pi \dot{\eta} \lambda \vartheta \sigma \mu \varepsilon \nu$; nun ist aber bekannt, daß der Nomophylax Joannes Eugenikos mit nach Italien gegangen ist. Freilich scheint mir diese Notiz (Nr. 85), die chronologisch allerletzte (1437), von einer anderen Hand, vermutlich von der des Sohnes Manuel, za stammen, die auch andere Notizen auf derselben Seite niedergeschrieben hat.

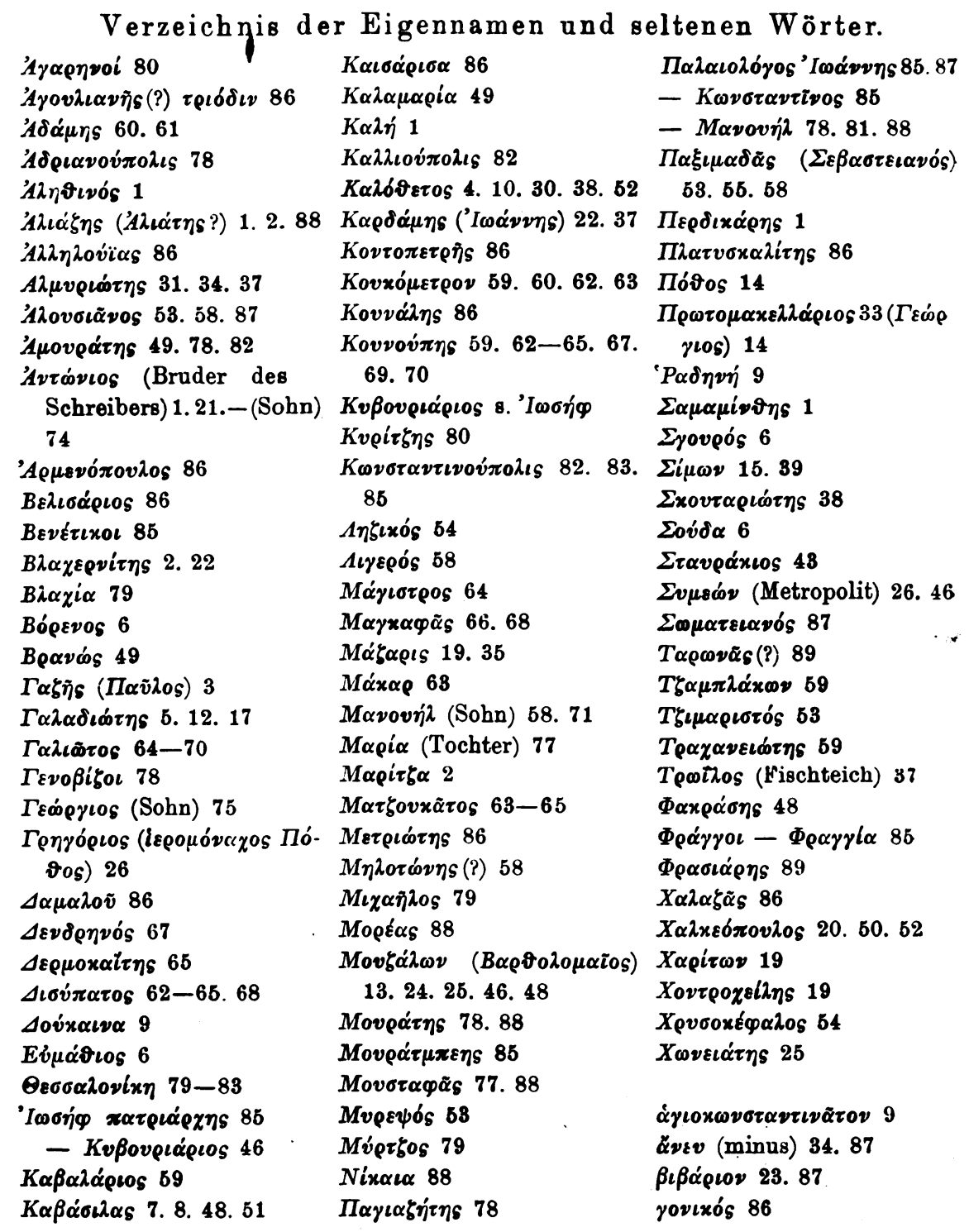


$\delta \alpha \nu \varepsilon \iota \alpha \times \alpha^{\prime} 87$

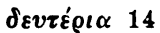

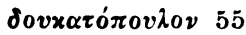

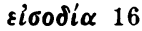

$\varepsilon \lambda \varepsilon \iota \pi \dot{\alpha} \sigma \vartheta \eta \nu \nu 1$

$\xi \xi \xi \mu \varepsilon \rho i \alpha$ 10. 31.87

$\dot{\xi} \xi \alpha \mu \eta v \alpha i 0 \nu$ 25. 33. 37. 51.

89

$\zeta \eta \tau o v ่ \lambda \iota v ~ 31.32 .42$

Athen. i $\sigma \alpha \sigma \mu \varepsilon \dot{v} 0 \mathrm{~s} 58$

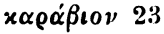

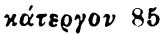

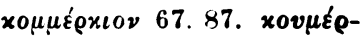
$x\llcorner\nu 87$

$\mu \alpha \lambda \alpha \gamma \mu \alpha^{\prime} \tau \iota \nu 0 S 9$

oxx $\alpha \mu \varepsilon p i c e \quad 48$

òdoxeovixá (Adverb) 85

ơ

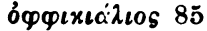

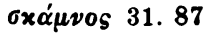

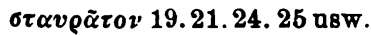

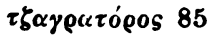

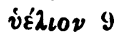

$\varphi \alpha \mu \iota \lambda i \alpha \quad 53$

$\varphi \lambda \omega \rho i o v ~ \beta \varepsilon v \varepsilon \tau น x o v 53$

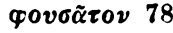

Sokr. Kugéas.

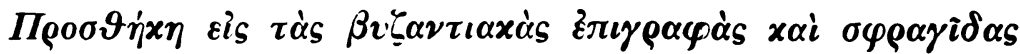
A $\lambda \mu v \rho o \tilde{v}$.

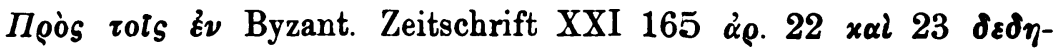

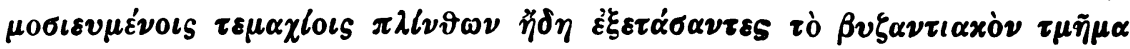
$\tau 0 \tilde{v} \mu 0 v \sigma \varepsilon l o v ' A \lambda \mu v \rho \circ \tilde{v} \varepsilon \tilde{v} \varrho 0 \mu \varepsilon \nu \pi \lambda \varepsilon i \sigma \tau \alpha$

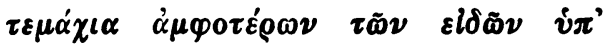

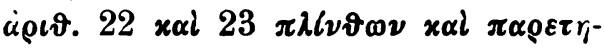

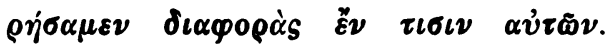

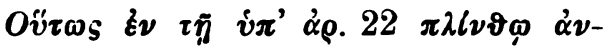

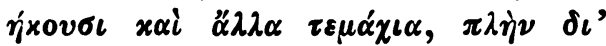

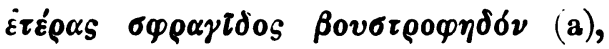

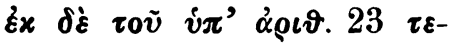

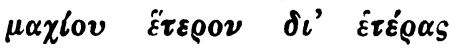
oqpajidos (b).

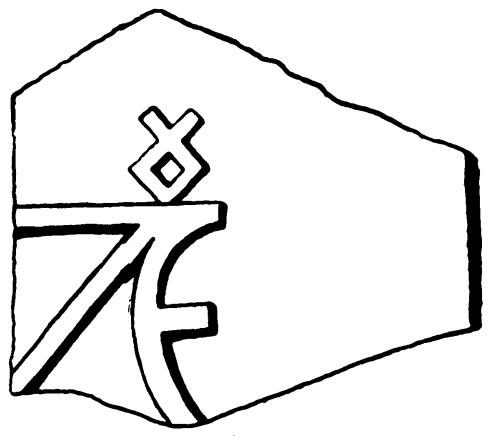

SХर्रत्र

$\Theta \mathrm{HB}^{8}$

a.

b.

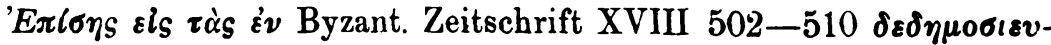

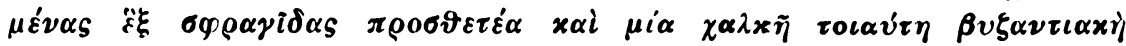

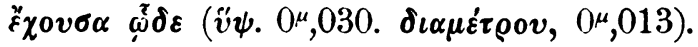

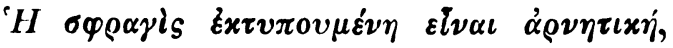

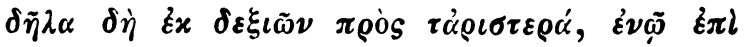

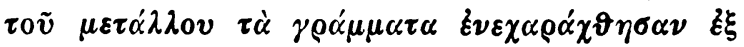

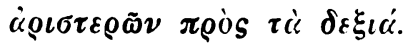

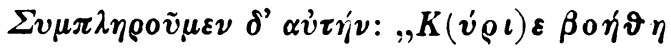
$N(\iota) x(\dot{\eta}) \tau(\alpha)^{"}$.

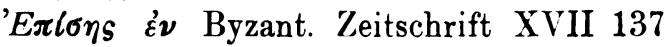

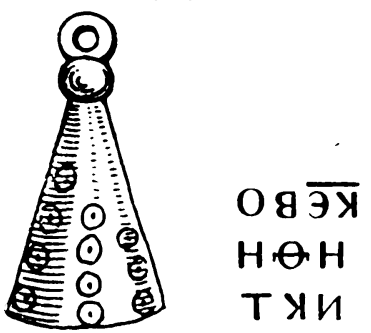

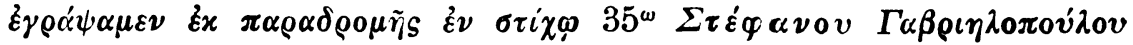

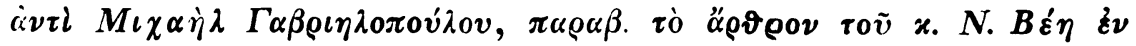
Byzant. Zeitschrift XXI 170.

'Ev'A $A \mu v \rho \tilde{\omega}$.

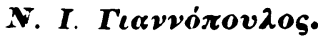

